\title{
The Uniqueness of the Integrated Density of States for the Schrödinger Operators for the Robin Boundary Conditions
}

\author{
By
}

Takuya Mine*

\begin{abstract}
The integrated density of states (IDS) for the Schrödinger operators is defined by using the eigenvalue counting function of the operator restricted to bounded regions with appropriate boundary conditions. Two sufficient conditions for the coincidence of the IDS for the Dirichlet boundary conditions and the IDS for the Robin boundary conditions are given. The proofs of some fundamental formulas, e.g. the change of variables, the chain rule and the divergence formula, for Lipschitz domains are given for the completeness.
\end{abstract}

\section{$\S 1$. Introduction}

\section{$\S 1.1$ Definition of the integrated density of states and results}

In this paper we shall consider the Schrödinger operators with magnetic fields in $d$-dimensional space:

$$
L=-\sum_{j=1}^{d}\left(\partial_{j}-i a_{j}\right)^{2}+V,
$$

where $\partial_{j}=\partial / \partial x_{j}, i=\sqrt{-1}$ and $a_{j}$ and $V$ are the multiplication operators by real-valued functions on $\boldsymbol{R}^{d}$. $\boldsymbol{a}=\left(a_{1}, \ldots, a_{d}\right)$ is called a (magnetic) vector

Communicated by T. Kawai. Received October 19, 2001. Revised December 3, 2001.

2000 Mathematics Subject Classification(s): Primary 35J10; Secondary 47N20, 81Q10.

Key words: Magnetic Schrödinger Operator, Integrated Density of States, Robin boundary conditions, Lipschitz domain.

* Division of Mathematics, Graduate School of Science, Kyoto University, Sakyo-ku, Kyoto 606-8502, Japan.

e-mail: mine@kusm.kyoto-u.ac.jp 
potential and $V$ a (electric) scalar potential. Throughout this paper we assume the following:

\section{Assumption 1.}

$$
\boldsymbol{a}=\left(a_{1}, \ldots, a_{d}\right) \in\left(L_{l o c}^{2}\left(\boldsymbol{R}^{d}\right)\right)^{d}, \quad V \in L_{l o c}^{1}\left(\boldsymbol{R}^{d}\right), \quad V \geq 0 .
$$

Under Assumption 1, $L$ can be defined by a quadratic form defined on $C_{0}^{\infty}\left(\boldsymbol{R}^{d}\right)$ (the space of compactly supported smooth functions on $\boldsymbol{R}^{d}$ ) and it is known that $L$ has a unique self-adjoint realization $H$ on $L^{2}\left(\boldsymbol{R}^{d}\right)$ whose form domain contains $C_{0}^{\infty}\left(\boldsymbol{R}^{d}\right)$ (see [I-K], [L-S]; see also Definition 1.4 below).

The integrated density of states (IDS) is a non-decreasing function $\rho^{\#}(\lambda)$ of $\lambda \in \boldsymbol{R}$ defined (formally) by

$$
\rho^{\#}(\lambda):=\lim _{\Omega \rightarrow \mathbf{R}^{d}, \Omega \in \mathcal{O}} \frac{N_{\Omega}^{\#}(\lambda)}{|\Omega|},
$$

if the limit exists. Here, $N_{\Omega}^{\#}(\lambda)$ is the number of eigenvalues less than or equal to $\lambda$ of the operator $L$ restricted to a bounded domain $\Omega$ with boundary conditions $(\#) .|\cdot|$ denotes the Lebesgue measure. $\mathcal{O}$ is a family of bounded open sets which is introduced to specify the way expanding $\Omega$ to the whole space $\boldsymbol{R}^{d}$ and we assume the following:

Assumption 2. $\mathcal{O}=\{\Omega\}$ is a family of bounded open sets and satisfies the following:

(A1) For every $N \in \boldsymbol{N}(:=\{1,2, \cdots\})$, there exists $\Omega \in \mathcal{O}$ such that $B_{N} \subset \Omega$.

(A2) As a function of $\Omega \in \mathcal{O}$,

$$
\frac{|\{x \in \Omega \mid \operatorname{dist}(x, \partial \Omega)<1\}|}{|\Omega|} \rightarrow 0 \quad \text { as } \Omega \rightarrow \boldsymbol{R}^{d} \text { in } \mathcal{O} \text {. }
$$

Here, $B_{R}$ is a open ball of radius $R$ centered at the origin and $\partial \Omega$ denotes the boundary of $\Omega$. The meaning of the notation as $\Omega \rightarrow \boldsymbol{R}^{d}$ in $\mathcal{O}$ is defined in the following:

Definition 1.1. For a function $F(\Omega)$ of $\Omega \in \mathcal{O}$, we say $F(\Omega) \rightarrow c$ as $\Omega \rightarrow \boldsymbol{R}^{d}$ in $\mathcal{O}$ if for every $\epsilon>0$ there exists $N_{0} \in \boldsymbol{N}$ such that $|F(\Omega)-c|<\epsilon$ for every $\Omega \in \mathcal{O}$ with $\Omega \supset B_{N_{0}}$.

For example, the family of all cubes (or balls) centered at the origin satisfies Assumption 2.

In this paper, we shall consider the following boundary conditions: 
(D) $\left.u\right|_{\partial \Omega}=0$.

(N) $\left.(\nabla-i \boldsymbol{a}) u\right|_{\partial \Omega} \cdot \boldsymbol{n}=0$.

(R) $\left.(\nabla-i \boldsymbol{a}) u\right|_{\partial \Omega} \cdot \boldsymbol{n}=-\left.\sigma_{\Omega} u\right|_{\partial \Omega}$.

Here, $\boldsymbol{n}$ denotes the unit outer normal vector on the boundary $\partial \Omega$ of $\Omega$. The function $\sigma_{\Omega}$ may depend on $\Omega \in \mathcal{O}$ and satisfies the following:

Assumption 3. $\sigma_{\Omega} \in L^{\infty}(\partial \Omega ; \boldsymbol{R})$ for every $\Omega \in \mathcal{O}$.

The letters $D, N, R$ correspond to the Dirichlet, Neumann and Robin boundary conditions, respectively. We denote their self-adjoint realizations $H_{\Omega}^{D}, H_{\Omega}^{N}$ and $H_{\Omega}^{R}$, respectively (see Definitions 1.4 and 1.5 below).

In this paper, we treat the uniqueness problem for the definition of IDS, that is, the problem of finding some sufficient conditions for the coincidence of the IDS for the Dirichlet and Robin boundary conditions.

There are several results which have proved the uniqueness of IDS for the magnetic Schrödinger operators for the Dirichlet and Neumann boundary conditions; see Nakamura [N], Hupfer-Leschke-Müller-Warzel [H-L-M-W1] (see also their preprint [H-L-M-W2]) and Doi-Iwatsuka-Mine [D-I-M] (there are several results in the non-magnetic case; see references in [D-I-M]). In particular, [D-I-M, Theorem 1.2] has proved that the limits $\rho^{D}$ and $\rho^{N}$ coincide with each other, in the weak topology of measures (see Definitions 1.2 and 1.3 below), under Assumptions 1, 2 and the following assumption:

Assumption 4. $\mathcal{O} \subset \operatorname{LM}(r, A, B)$ for some $r, A, B>0$.

Roughly speaking, Assumption 4 means that the boundary $\partial \Omega$ of $\Omega \in \mathcal{O}$ is represented by Lipschitz continuous functions whose Lipschitz constants are uniformly bounded (see Section 1.3 below, for the definition of $\operatorname{LM}(r, A, B)$ ). This result ([D-I-M, Theorem 1.2]) also implies that, if another boundary conditions (\#) satisfy $H_{\Omega}^{D} \geq H_{\Omega}^{\#} \geq H_{\Omega}^{N}$ in the form sense, then the limits $\rho^{\#}, \rho^{D}$ and $\rho^{N}$ exist and coincide with each other in the weak topology of measures, when one of them exists. For example, the periodic conditions, the (magnetic) Bloch wave conditions and the Dirichlet-Neumann mixed conditions are included in this case. This fact is easily proved by the inequality

$$
N_{\Omega}^{D}(\lambda) \leq N_{\Omega}^{\#}(\lambda) \leq N_{\Omega}^{N}(\lambda)
$$

for every $\lambda \in \boldsymbol{R}$, which immediately follows from the min-max principle. 
However, the Robin boundary conditions are not included in this case, when the boundary function $\sigma_{\Omega}$ has nonzero negative part. To see this, notice that the quadratic form associated to the operator $H_{\Omega}^{R}$ is the following (see Section 1.3):

$$
\left(H_{\Omega}^{R} u, u\right)_{\Omega}=\|(\nabla-i \boldsymbol{a}) u\|_{\Omega}^{2}+\left\|V^{1 / 2} u\right\|_{\Omega}^{2}+\int_{\partial \Omega} \sigma_{\Omega}|u|^{2} d S .
$$

Here, $\|\cdot\|_{\Omega}$ denotes the $L^{2}(\Omega)$-norm and $d S$ the $(d-1)$-dimensional surface measure. (1.3) and the min-max principle show that, for a fixed energy $\lambda$ and a fixed region $\Omega$, the value $N_{\Omega}^{R}(\lambda)$ increases as $\sigma_{\Omega}$ decreases. Notice also that, the Neumann boundary conditions are particular cases of the Robin boundary conditions $\left(\sigma_{\Omega}=0\right)$.

In this paper, we will give two sufficient conditions which guarantee the limit $\rho^{D}$ and $\rho^{R}$ coincide with each other, in the weak topology of measures. From the above consideration, we should require some restriction on the growth of the negative part of $\sigma_{\Omega}$ as a function of $\Omega \in \mathcal{O}$, in addition to Assumptions 1, 2 and 4. We consider following two assumptions on $\sigma_{\Omega}^{-}:=\max \left(\operatorname{ess} \cdot \sup \left(-\sigma_{\Omega}\right)\right.$, $0)$ :

Assumption 5. $\quad\left(\sigma_{\Omega}^{-}\right)^{d}|\partial \Omega|^{\prime} /|\Omega| \rightarrow 0$ as $\Omega \rightarrow \boldsymbol{R}^{d}$ in $\mathcal{O}$.

Assumption 6. $\quad\left(\sigma_{\Omega}^{-}\right)^{d-1}|\partial \Omega|^{\prime} /|\Omega| \rightarrow 0$ as $\Omega \rightarrow \boldsymbol{R}^{d}$ in $\mathcal{O}$.

Here, $|\partial \Omega|^{\prime}$ denotes the surface volume of $\partial \Omega$. Under Assumption 4, there exists a constant $C=C(r, A, B, d)>1$ such that

$$
C^{-1}|\partial \Omega|^{\prime}<|\{x \mid \operatorname{dist}(x, \partial \Omega)<1\}|<C|\partial \Omega|^{\prime}
$$

(see [D-I-M, Proposition 4.5]). Hence, Assumption 2 is equivalent to the condition $|\partial \Omega|^{\prime} /|\Omega| \rightarrow 0$ as $\Omega \rightarrow \boldsymbol{R}^{d}$ in $\mathcal{O}$ under Assumption 4 . Thus, we see that Assumption 6 is weaker than Assumption 5 under Assumption 4.

For describing the precise statement of the main theorem, we review some definitions used in [D-I-M]. The operator $H_{\Omega}^{\#}(\#=D, N, R)$ has a compact resolvent under the assumption $\Omega \in L M(r, A, B)$ for some $r, A, B>0$ (see [D-I-M, Theorem 6.2] and Theorem 1.2 below). Hence $H_{\Omega}^{\#}$ has a complete orthonormal system of eigenfunctions.

Definition 1.2. Let $\Omega \in L M(r, A, B)$ for some $r, A, B>0$. Let $C_{0}(\boldsymbol{R})$ be the space of all compactly supported continuous functions on $\boldsymbol{R}$. For $\#=D$, $N, R$, define a linear functional $\rho_{\Omega}^{\#}$ by

$$
\rho_{\Omega}^{\#}(f):=\frac{\operatorname{tr}\left(f\left(H_{\Omega}^{\#}\right)\right)}{|\Omega|}=\int_{\mathbf{R}} f(\lambda) d \rho_{\Omega}^{\#}(\lambda)
$$


for $f \in C_{0}(\boldsymbol{R})$. Here, $f\left(H_{\Omega}^{\#}\right)$ denotes the operator defined by the functional calculus and $\operatorname{tr}(K)$ denotes the trace of a trace class operator $K$. The measure $d \rho_{\Omega}^{\#}$ is defined by

$$
d \rho_{\Omega}^{\#}=\sum_{n=1}^{\infty} \delta_{\lambda_{n}^{\#},}
$$

where $\lambda_{n}^{\#}$ is the $n$-th eigenvalue (from below, counting multiplicity) of $H_{\Omega}^{\#}$ and $\delta_{\lambda}$ is the Dirac measure concentrated on $\lambda$.

Using this notation, we can rewrite the right hand side of (1.1) as

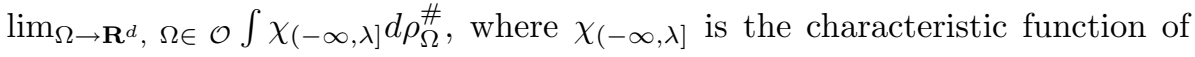
the interval $(-\infty, \lambda]$. To describe our main theorems, we use a continuous function $f$ in place of the discontinuous function $\chi_{(-\infty, \lambda]}$. It enables us to describe the statement of main theorems in terms of the weak topology of measures.

Definition 1.3. Let $d \rho_{\Omega}^{\#}(\#=D, N, R)$ as Definition 1.2 and $d \rho^{\#}$ some Borel measure on $\boldsymbol{R}$. We say $d \rho_{\Omega}^{\#} \rightarrow d \rho^{\#}$ as $\Omega \rightarrow \boldsymbol{R}^{d}$ in $\mathcal{O}$ if

$$
\int_{\mathbf{R}} f d \rho_{\Omega}^{\#} \rightarrow \int_{\mathbf{R}} f d \rho^{\#} \quad \text { as } \quad \Omega \rightarrow \boldsymbol{R}^{d} \text { in } \mathcal{O},
$$

for every $f \in C_{0}(\boldsymbol{R})$.

Consider the following statements:

$(\text { Existence })^{\mathrm{D}}$ There exists a Borel measure $d \rho^{D}$ such that $d \rho_{\Omega}^{D} \rightarrow d \rho^{D}$ as $\Omega \rightarrow$ $\boldsymbol{R}^{d}$ in $\mathcal{O}$.

$(\text { Existence })^{\mathrm{R}}$ There exists a Borel measure $d \rho^{R}$ such that $d \rho_{\Omega}^{R} \rightarrow d \rho^{R}$ as $\Omega \rightarrow$ $\boldsymbol{R}^{d}$ in $\mathcal{O}$.

Our first result is the following:

Theorem 1.1. Suppose that Assumptions 1 through 5 hold. Then, the statement $(\text { Existence })^{\mathrm{D}}$ and $(\text { Existence })^{\mathrm{R}}$ are equivalent to each other. Moreover, if one of them holds, the limit measures $d \rho^{D}$ and $d \rho^{R}$ coincide. In particular, the measure $d \rho^{R}$ is independent of the choice of the family $\left\{\sigma_{\Omega}\right\}$ satisfying Assumption 5.

If we use the discontinuous function $\chi_{(-\infty, \lambda]}$ in place of $f \in C_{0}(\boldsymbol{R})$, we get the result that the limit functions $\rho^{D}(\lambda)$ and $\rho^{R}(\lambda)$ coincide at the continuity point $\lambda$ of $\rho^{D}(\cdot)$ (or $\rho^{R}(\cdot)$ ), when one of them exists. The function $\rho^{\#}$ is 
monotone nondecreasing and hence the discontinuity points of $\rho^{D}(\cdot)\left(\rho^{R}(\cdot)\right)$ are at most countable.

Theorem 1.1 is an extension of [D-I-M, Theorem 1.2]. Theorem 1.1 follows from the next estimate:

Theorem 1.2. $\quad$ Suppose Assumption 1 holds. Let $r, A, B>0, \Omega \in$ $L M(r, A, B)$ and $\sigma \in L^{\infty}(\partial \Omega ; \boldsymbol{R}) . C_{2}=C_{2}(r, A, B, d)>0$ is a constant given in (ii) of Proposition 3.2. Then, there exists constants $C>0$ and $C_{0}>0$, dependent only on $r, A, B, d$ such that

$$
N_{\Omega}^{R}(\lambda) \leq N_{\Omega}^{D}(\lambda+\epsilon)+C\left(\epsilon^{-d / 2}\left(\lambda+C_{0}\right)_{+}^{d / 2}+\left(\lambda+C_{0}+M_{0}\left(\sigma^{-}\right)^{2}\right)_{+}^{d / 2}\right)|\partial \Omega|^{\prime}
$$

for every $0<\epsilon<1, \Omega \in L M(r, A, B)$ and $\lambda \in \boldsymbol{R}$. Here, $M_{0}=2 C_{2}^{2},(x)_{+}:=$ $\max (x, 0)$ for $x \in \boldsymbol{R}$ and

$$
\sigma^{-}:=\max \left(\operatorname{ess} \cdot \sup (-\sigma), 1 /\left(r C_{2}\right)\right) .
$$

In particular, $H_{\Omega}^{R}$ has a compact resolvent for every $\Omega \in L M(r, A, B)$.

We can deduce Theorem 1.1 from Theorem 1.2 by the same argument used in [D-I-M, Proof of Theorem 1.2 assuming Theorem 6.2] and hence we omit its proof.

We can see, however, that the growth order of $\sigma_{\Omega}^{-}$is not optimal, at least when $\boldsymbol{a}=0$ :

Theorem 1.3. Suppose that Assumptions 1, 2, 3, 4 and 6 hold and $\boldsymbol{a}=0$. Then, the conclusion of Theorem 1.1 with Assumption 5 replaced by Assumption 6 holds.

Theorem 1.3 follows from the next estimate and we omit the proof of Theorem 1.3:

Theorem 1.4. Suppose $\boldsymbol{a}=0$ and $V \in L_{\text {loc }}^{1}\left(\boldsymbol{R}^{d} ; \boldsymbol{R}\right), V \geq 0$. Let $r, A, B>0, \Omega \in L M(r, A, B)$ and $\sigma \in L^{\infty}(\partial \Omega ; \boldsymbol{R})$. Then, there exist constants $C>0$ and $C_{0}>0$ dependent only on $r, A, B, d$ and a constant $M_{0}>1$ dependent only on $A, d$ such that

$$
\begin{aligned}
N_{\Omega}^{R}(\lambda) \leq & N_{\Omega}^{D}(\lambda+\epsilon) \\
& +C\left(\epsilon^{-d / 2}\left(\lambda+C_{0}\right)_{+}^{d / 2}+\left(\lambda+C_{0}+M_{0}\left(\sigma^{-}\right)^{2}\right)_{+}^{(d-1) / 2}\right)|\partial \Omega|^{\prime}
\end{aligned}
$$

for every $0<\epsilon<1, \Omega \in L M(r, A, B)$ and $\lambda \in \boldsymbol{R}$. Here,

$$
\sigma^{-}=\max \left(\operatorname{ess} \cdot \sup (-\sigma), \sqrt{1+A^{2}} /\left(M_{0} r\right)\right) .
$$


We have organized this paper as follows. In Section 1.2, we define the self-adjoint realizations $H_{\Omega}^{D}, H_{\Omega}^{N}$ and $H_{\Omega}^{R}$. In Section 1.3 , we define a class of bounded open sets with Lipschitz boundary, $\operatorname{LM}(r, A, B)$. In Section 2.1, we prove Theorem 1.2. The proof of Theorem 1.2 is parallel to that of [DI-M, Theorem 6.2] except for the use of the magnetic trace inequality. The difference between the Neumann conditions and the Robin conditions is the boundary term of their quadratic forms, which is estimated by the magnetic trace inequality. In Section 2.2, we prove Theorem 1.4. In the proof of Theorem 1.4, we do not use the trace inequality for the estimation of boundary terms, but the direct computation of eigenvalues using separation of variables (which also proves that the growth order in Assumption 6 is actually optimal). The latter method cannot be applied to the case in the presence of the magnetic potential $\boldsymbol{a}$. At present, it is not known whether the estimate (1.6) holds for general $\boldsymbol{a} \in L_{\text {loc }}^{2}\left(\boldsymbol{R}^{d}\right)$. In Section 3, we prove the magnetic trace inequality, i.e., an estimate of the operator norm of the restriction operator $\left.\cdot\right|_{\partial \Omega}$ from the magnetic Sobolev space $W_{a, V}^{1}(\Omega)$ (see Definition 1.4 below) to $L^{2}(\partial \Omega, d S)$. In Section 4 , we prove several fundamental formulas, e.g., the change of variables, the chain rule and the divergence formula, for Lipschitz domains, for the sake of completeness.

\section{$\S 1.2$. Self-adjoint realizations}

Let us define the self-adjoint realizations $H_{\Omega}^{D}, H_{\Omega}^{N}$ and $H_{\Omega}^{R}$, in terms of quadratic forms. In the sequel, $\nabla$ denotes the distributional gradient, that is, $\nabla u=\left(\partial_{1} u, \ldots, \partial_{d} u\right) \in\left(\mathcal{D}^{\prime}\right)^{d}$, for $u \in \mathcal{D}^{\prime}$ (the space of the Schwartz distributions, see [Ru2]), $Q(h)$ the form domain of a sesqui-linear form $h$ and $D(A)$ the operator domain of a self-adjoint operator $A$. For $u, v \in L^{2}(\Omega)$, we denote the inner product $(u, v)_{\Omega}:=\int_{\Omega} \bar{u} v$ and the norm $\|u\|_{\Omega}^{2}:=(u, u)_{\Omega}$. Formally, we have by integration by parts

$$
\begin{aligned}
& \left(-(\nabla-i \boldsymbol{a})^{2} u+V u, v\right)_{\Omega} \\
& \quad=((\nabla-i \boldsymbol{a}) u,(\nabla-i \boldsymbol{a}) v)_{\Omega}+\left(V^{1 / 2} u, V^{1 / 2} v\right)_{\Omega}+\int_{\partial \Omega} \overline{(\nabla-i \boldsymbol{a}) u \cdot \boldsymbol{n}} v d S,
\end{aligned}
$$

where $d S$ denotes the surface measure on $\partial \Omega$. From this equality, we see that the boundary term $\int_{\partial \Omega} \overline{(\nabla-i \boldsymbol{a}) u \cdot \boldsymbol{n}} v$ vanishes when both $u$ and $v$ satisfy the boundary conditions $(D)$ (or $(N)$ ). Thus, we can define the operators $H_{\Omega}^{D}$ and $H_{\Omega}^{N}$ without any assumption on the boundary $\partial \Omega$ : 
Definition 1.4. Let $\Omega$ be an open subset of $\boldsymbol{R}^{d}$ and let $\boldsymbol{a} \in\left(L^{2}(\Omega ; \boldsymbol{R})\right)^{d}$, $V \in L^{1}(\Omega ; \boldsymbol{R}), V \geq 0$. Define a subspace $W_{a, V}^{1}(\Omega)$ of $L^{2}(\Omega)$ by

$$
W_{a, V}^{1}(\Omega)=\left\{u \in L^{2}(\Omega) \mid(\nabla-i \boldsymbol{a}) u \in\left(L^{2}(\Omega)\right)^{d}, V^{1 / 2} u \in L^{2}(\Omega)\right\} .
$$

For $u, v \in W_{a, V}^{1}(\Omega)$, define a sesqui-linear form $h_{a, V}(u, v)$ by

$$
h_{a, V}(u, v):=((\nabla-i \boldsymbol{a}) u,(\nabla-i \boldsymbol{a}) v)_{\Omega}+\left(V^{1 / 2} u, V^{1 / 2} v\right)_{\Omega} .
$$

The space $W_{a, V}^{1}(\Omega)$ is a Hilbert space equipped with the norm $\|u\|_{a, V}^{2}:=$ $h_{a, V}(u, u)+\|u\|_{\Omega}^{2}$. Let $W_{0, a, V}^{1}(\Omega)$ be the closure of $C_{0}^{\infty}(\Omega)$ with respect to the norm $\|\cdot\|_{a, V}$. Define the form domains $Q\left(h_{\Omega}^{D}\right):=W_{0, a, V}^{1}(\Omega), Q\left(h_{\Omega}^{N}\right):=$ $W_{a, V}^{1}(\Omega)$ and define the sesqui-linear forms $h_{\Omega}^{\#}(\#=D$ or $N)$ by

$$
h_{\Omega}^{\#}(u, v):=h_{a, V}(u, v),
$$

for $u, v \in Q\left(h_{\Omega}^{\#}\right)$. Denote the corresponding quadratic form by $h_{\Omega}^{\#}[u]=$ $h_{\Omega}^{\#}(u, u)$. The quadratic form $h_{\Omega}^{\#}$ is closed and non-negative, and we can define a self-adjoint operator $H_{\Omega}^{\#}$ associated with $h_{\Omega}^{\#}$ by the relation

$$
\left(H_{\Omega}^{\#} u, v\right)_{\Omega}=h_{\Omega}^{\#}(u, v)
$$

for every $u \in D\left(H_{\Omega}^{\#}\right)$ and every $v \in Q\left(h_{\Omega}^{\#}\right)($ see $[\mathrm{K}])$.

In the case of the Robin boundary conditions, the boundary term in (1.7) is present. To define the restriction $\left.u\right|_{\partial \Omega}$ of a function $u$ on $\Omega$, we need some smoothness assumption on $u$ and some regularity assumption on the boundary $\partial \Omega$. A sufficient condition is $u \in W_{a, V}^{1}(\Omega)$ and $\Omega \in L M(r, A, B)$. When $\boldsymbol{a}=0$, this condition is well-known (in fact, the restriction operator is a bounded operator from $W^{1}(\Omega)$ to $W^{1 / 2}(\partial \Omega)$; see [Wl]). However, we cannot find the proof of this fact in more general case $\boldsymbol{a} \in L^{2}(\Omega)$. In Proposition 3.2, we will prove that the restriction operator $\left.\right|_{\partial \Omega}$ is bounded from $W_{a, V}^{1}(\Omega)$ to $L^{2}(\partial \Omega)$, under the assumptions $\boldsymbol{a} \in L^{2}(\Omega), V \in L^{1}(\Omega), V \geq 0$ and $\Omega \in L M(r, A, B)$ for some $r, A, B>0$. Thus, we define the self-adjoint realization $H_{\Omega}^{R}$ by the following:

Definition 1.5. Let $\Omega \in \operatorname{LM}(r, A, B)$ for some $r, A, B>0$. Let $\boldsymbol{a} \in$ $\left(L^{2}(\Omega ; \boldsymbol{R})\right)^{d}, V \in L^{1}(\Omega ; \boldsymbol{R}), V \geq 0$ and $\sigma \in L^{\infty}(\partial \Omega ; \boldsymbol{R})$. Define a closed sesqui-linear form $h_{\Omega}^{R}$ by

$$
h_{\Omega}^{R}(u, v)=h_{a, V}(u, v)+(\sigma u, v)_{\partial \Omega}
$$


for $u, v \in Q\left(h_{\Omega}^{R}\right):=W_{a, V}^{1}(\Omega)$, where $(u, v)_{\partial \Omega}:=\int_{\partial \Omega} \bar{u} v d S$ (we often abbreviate the function $\left.u\right|_{\partial \Omega}$ restricted to the boundary simply as $u$, when no confusion may occur). Denote the corresponding quadratic form by $h_{\Omega}^{R}[u]=h_{\Omega}^{R}(u, u)$. Define a self-adjoint operator $H_{\Omega}^{R}$ associated with $h_{\Omega}^{R}$ by the relation (1.9).

\section{$\S 1.3 . \quad$ Definition of $\operatorname{LM}(r, A, B)$}

In this subsection, we shall remind the definition of manifolds with Lipschitz boundary $L M(r, A, B)(r, A, B>0)$, which has been introduced in [D-I$\mathrm{M}]$. In the sequel, we use the notation $x=\left(x^{\prime}, x_{d}\right)$ for $x \in \boldsymbol{R}^{d}$, where $x^{\prime} \in \boldsymbol{R}^{d-1}$, $x_{d} \in \boldsymbol{R}$.

Definition 1.6. Let $r, A, B>0$. For a bounded open set $\Omega$, we say $\Omega \in$ $L M(r, A, B)$ if there exists an integer $K>0$ and a system $\left\{U_{k}, \chi_{k}, S_{k}, \phi_{k}\right\}_{k=1}^{K}$ satisfying (i)-(v) below:

(i) $U_{k}$ is a bounded open set in $\boldsymbol{R}^{d} \cdot \chi_{k}$ is a congruent transformation in $\boldsymbol{R}^{d}$, which is written as $\chi_{k}(x)=A_{k} x+a_{k}$, where $A_{k}$ is an orthogonal matrix and $a_{k}$ is a constant vector. $S_{k}$ is a rectangle in $\boldsymbol{R}^{d-1}$. For $t>0$, we define

$$
S_{k}(t):=\left\{x^{\prime} \in \boldsymbol{R}^{d-1} \mid \operatorname{dist}\left(x^{\prime}, S_{k}\right)<t\right\} .
$$

$\phi_{k} \in \operatorname{Lip}\left(\overline{S_{k}(r)} ; \boldsymbol{R}\right)$, the space of all real-valued Lipschitz continuous functions on $\overline{S_{k}(r)}$.

(ii) For $k=1, \ldots, K$,

$$
\begin{aligned}
\chi_{k}\left(U_{k}\right) & =\left\{\left(x^{\prime}, x_{d}\right) \in \boldsymbol{R}^{d} \mid x^{\prime} \in S_{k}(r), \phi_{k}\left(x^{\prime}\right)-r<x_{d}<\phi_{k}\left(x^{\prime}\right)+r\right\} \\
\chi_{k}\left(U_{k} \cap \Omega\right) & =\left\{\left(x^{\prime}, x_{d}\right) \in \boldsymbol{R}^{d} \mid x^{\prime} \in S_{k}(r), \phi_{k}\left(x^{\prime}\right)-r<x_{d}<\phi_{k}\left(x^{\prime}\right)\right\} \\
\chi_{k}\left(U_{k} \cap \partial \Omega\right) & =\left\{\left(x^{\prime}, x_{d}\right) \in \boldsymbol{R}^{d} \mid x^{\prime} \in S_{k}(r), x_{d}=\phi_{k}\left(x^{\prime}\right)\right\}
\end{aligned}
$$

(iii) $\partial \Omega \subset \cup_{k=1}^{K} \chi_{k}^{-1}\left\{\left(x^{\prime}, x_{d}\right) \in \boldsymbol{R}^{d} \mid x^{\prime} \in S_{k}, x_{d}=\phi_{k}\left(x^{\prime}\right)\right\}$.

(iv) $\left\|\nabla \phi_{k}\right\|_{\infty} \leq A$, for $k=1, \ldots, K$.

(v) $\#\left\{k \mid x \in U_{k}\right\} \leq B$, for every $x \in \boldsymbol{R}^{d}$.

We write also $\left(\Omega,\left\{U_{k}, \chi_{k}, S_{k}, \phi_{k}\right\}_{k=1}^{K}\right) \in L M(r, A, B)$ to mean that $\Omega \in$ $L M(r, A, B)$ and $\left\{U_{k}, \chi_{k}, S_{k}, \phi_{k}\right\}_{k=1}^{K}$ is a system satisfying the properties (i)(v) above. 


\section{$\S 2 . \quad$ Proof of Main Theorems}

\section{$\S 2.1$. Proof of Theorem 1.2}

In this subsection, we shall prove Theorem 1.2. The proof of Theorem 1.2 is parallel to that of [D-I-M, Theorem 6.2], except for the estimation of the boundary term. So we use some notations and results used in [D-I-M].

Proof of Theorem 1.2. Let $r, A, B>0,\left(\Omega,\left\{U_{k}, \chi_{k}, S_{k}, \phi_{k}\right\}_{k=1}^{K}\right) \in L M$ $(r, A, B)$ and $\sigma \in L^{\infty}(\partial \Omega ; \boldsymbol{R})$. Take a large number $R>0$. We take two partitions of unity $\left\{\alpha_{j}\right\}_{j=1,2}$ and $\left\{\beta_{k}\right\}_{k=0, \ldots, K}$ given in [D-I-M, Propositions 4.3 and 4.4]. The partition $\left\{\alpha_{j}\right\}_{j=1,2}$ satisfies

$$
\begin{aligned}
& \alpha_{1}, \quad \alpha_{2} \in C^{\infty}\left(\boldsymbol{R}^{d}\right), \quad \alpha_{j} \geq 0, \quad\left\|\nabla \alpha_{j}\right\|_{\infty} \leq M_{1} / R \quad(j=1,2), \\
& \alpha_{1}^{2}+\alpha_{2}^{2} \equiv 1 \text { on } R^{d}, \quad \operatorname{supp} \alpha_{1} \subset \Omega \backslash \overline{(\partial \Omega)_{R / 2}}, \quad \operatorname{supp} \alpha_{2} \subset\left(\Omega^{c}\right)_{R},
\end{aligned}
$$

where $M_{1}=M_{1}(d)$ is a positive constant and $(X)_{R}=\left\{x \in \boldsymbol{R}^{d} \mid \operatorname{dist}(x, X)<R\right\}$ for a subset $X$ of $\boldsymbol{R}^{d}$. The partition $\left\{\beta_{k}\right\}_{k=0, \ldots, K}$ satisfies

$$
\begin{aligned}
& \beta_{k} \in C^{\infty}\left(\boldsymbol{R}^{d}\right), \quad \beta_{k} \geq 0 \quad(k=0, \ldots, K), \\
& \sum_{k=0}^{K} \beta_{k}^{2}=1 \quad \text { on some neighborhood of } \bar{\Omega}, \\
& \operatorname{supp} \beta_{0} \subset \Omega \backslash \overline{(\partial \Omega)_{r_{0}}}, \quad \operatorname{supp} \beta_{k} \subset U_{k} \quad(\text { for } k=1, \ldots, K), \\
& \sum_{k=0}^{K}\left|\nabla \beta_{k}\right|^{2} \leq M_{2} \frac{(1+A)^{2}(1+B)}{r^{2}} \quad \text { on } \boldsymbol{R}^{d},
\end{aligned}
$$

where $r_{0}=r /\{100(A+1)\}$ and $M_{2}=M_{2}(d)$ is a positive constant.

Take $u \in Q\left(h_{\Omega}^{R}\right)$. Since $\alpha_{1}^{2}+\alpha_{2}^{2}=1$, we have

$$
h_{\Omega}^{R}[u]=\sum_{j=1}^{2}\left(\left\|\alpha_{j}(\nabla-i \boldsymbol{a}) u\right\|_{\Omega}^{2}+\left\|\alpha_{j} V^{1 / 2} u\right\|_{\Omega}^{2}\right)+\left(\sigma \alpha_{2} u, \alpha_{2} u\right)_{\partial \Omega} .
$$

By a simple computation (see [C-F-K-S, Theorem 3.2]), we have

$$
\begin{aligned}
& \left\|\alpha_{j}(\nabla-i \boldsymbol{a}) u\right\|_{\Omega}^{2} \\
& \quad=\left\|(\nabla-i \boldsymbol{a})\left(\alpha_{j} u\right)\right\|_{\Omega}^{2}-\left\|\left(\nabla \alpha_{j}\right) u\right\|_{\Omega}^{2}-\operatorname{Re}\left(\nabla\left(\alpha_{j}^{2}\right) \cdot \nabla u, u\right)_{\Omega} .
\end{aligned}
$$

From (2.3) and (2.4), we have

$$
\begin{aligned}
h_{\Omega}^{R}[u]= & \sum_{j=1}^{2}\left(\left\|(\nabla-i \boldsymbol{a})\left(\alpha_{j} u\right)\right\|_{\Omega}^{2}-\left\|\left(\nabla \alpha_{j}\right) u\right\|_{\Omega}^{2}+\left\|V^{1 / 2}\left(\alpha_{j} u\right)\right\|_{\Omega}^{2}\right) \\
& +\left(\sigma \alpha_{2} u, \alpha_{2} u\right)_{\partial \Omega},
\end{aligned}
$$


where we used $\nabla\left(\alpha_{1}^{2}+\alpha_{2}^{2}\right)=0$. Since supp $\alpha_{1} u$ is a compact set in $\Omega$, we have $\alpha_{1} u \in Q\left(h_{\Omega}^{D}\right)$ by (v) of [D-I-M, Proposition 2.1]. Hence we have by (2.1) and $(2.5)$

$$
h_{\Omega}^{R}[u] \geq\left(h_{\Omega}^{D}-\epsilon_{R}\right)\left[\alpha_{1} u\right]+\left(h_{\Omega}^{R}-\epsilon_{R}\right)\left[\alpha_{2} u\right],
$$

where $\epsilon_{R}:=2 M_{1}^{2} / R^{2}$.

Put $\gamma_{k}=\alpha_{2} \beta_{k}$ for $k=0, \ldots, K$. Since $\sum \beta_{k}^{2}=1$, we have similarly by $(2.2)$

$$
h_{\Omega}^{R}\left[\alpha_{2} u\right] \geq \sum_{k=0}^{K}\left(h_{\Omega}^{R}-C_{1}\right)\left[\gamma_{k} u\right],
$$

where $C_{1}=M_{2}(1+A)^{2}(1+B) / r^{2}$. Since $\operatorname{supp} \gamma_{0} u$ is a compact set in $\Omega \cap$ $(\partial \Omega)_{R}$, we have $\gamma_{0} u \in Q\left(h_{\Omega \cap(\partial \Omega)_{R}}^{D}\right)$ by (v) of [D-I-M, Proposition 2.1]. For $k=1, \ldots, K, \operatorname{supp} \gamma_{k} u \subset U_{k}^{-}:=U_{k} \cap \Omega$. Thus, we obtain by (2.6) and (2.7)

$$
\begin{aligned}
h_{\Omega}^{R}[u] \geq & \left(h_{\Omega}^{D}-\epsilon_{R}\right)\left[\alpha_{1} u\right]+\left(h_{\Omega \cap(\partial \Omega)_{R}}^{D}-C_{1}-\epsilon_{R}\right)\left[\gamma_{0} u\right] \\
& +\sum_{k=1}^{K}\left(h_{U_{k}^{-}}^{R}-C_{1}-\epsilon_{R}\right)\left[\gamma_{k} u\right] .
\end{aligned}
$$

By (ii) of Proposition 3.2, we have

$$
\begin{aligned}
\left(\sigma \gamma_{k} u, \gamma_{k} u\right)_{\partial \Omega} & \geq-\sigma^{-}\left\|\gamma_{k} u\right\|_{\partial \Omega}^{2} \\
& \geq-C_{2}\left(\epsilon_{1}^{-1} \sigma^{-}\left\|\gamma_{k} u\right\|_{U_{k}^{-}}^{2}+\epsilon_{1} \sigma^{-}\left\|(\nabla-i \boldsymbol{a})\left(\gamma_{k} u\right)\right\|_{U_{k}^{-}}^{2}\right) \\
& \geq-C_{2}\left(\epsilon_{1}^{-1} \sigma^{-}\left\|\gamma_{k} u\right\|_{U_{k}^{-}}^{2}+\epsilon_{1} \sigma^{-} h_{U_{k}^{-}}^{N}\left[\gamma_{k} u\right]\right)
\end{aligned}
$$

for every $\epsilon_{1}$ with $r>\epsilon_{1}>0$, where $\sigma^{-}:=\max \left(\operatorname{ess} \sup \{-\sigma\}, 1 /\left(r C_{2}\right)\right)$ and $C_{2}>0$ is a constant dependent only on $r, A, B$, $d$. Take $\epsilon_{1}:=1 /\left(2 C_{2} \sigma^{-}\right)$. Note that $\epsilon_{1}<r$. Then, we have by (2.8) and (2.9),

$$
\begin{aligned}
h_{\Omega}^{R}[u] \geq & \left(h_{\Omega}^{D}-\epsilon_{R}\right)\left[\alpha_{1} u\right]+\left(h_{\Omega \cap(\partial \Omega)_{R}}^{D}-C_{1}-\epsilon_{R}\right)\left[\gamma_{0} u\right] \\
& +\sum_{k=1}^{K}\left(\frac{1}{2} h_{U_{k}^{-}}^{N}-C_{1}-2 C_{2}^{2}\left(\sigma^{-}\right)^{2}-\epsilon_{R}\right)\left[\gamma_{k} u\right] .
\end{aligned}
$$

Next, we repeat the extension argument given in the proof of [D-I-M, Theorem 6.2] (see (6.17)-(6.21) in [D-I-M]). As a result, we obtain an extended vector potential $\widetilde{\boldsymbol{a}_{k}}$ and an extended function $\widetilde{\gamma_{k} u}$ defined on $U_{k}$, which satisfy $\left.\widetilde{\boldsymbol{a}_{k}}\right|_{U_{k}^{-}}=\left.\boldsymbol{a}\right|_{U_{k}^{-}},\left.\widetilde{\gamma_{k} u}\right|_{U_{k}^{-}}=\left.\gamma_{k} u\right|_{U_{k}^{-}}, \widetilde{\gamma_{k} u} \in W_{0, \widetilde{a_{k}}, 0}^{1}\left(U_{k}\right)$ and

$$
\begin{aligned}
& \left\|\widetilde{\gamma_{k} u}\right\|_{U_{k}}^{2}=2\left\|\gamma_{k} u\right\|_{U_{k}^{-}}^{2}, \\
& h_{U_{k}^{-}}^{N}\left[\gamma_{k} u\right] \geq \frac{1}{C_{3}} h_{k}^{D}\left[\frac{1}{\sqrt{2}} \widetilde{\gamma_{k} u}\right] .
\end{aligned}
$$


Here, $C_{3}=2 A^{2}+A+1$ and $h_{k}^{D}$ is a quadratic form on $L^{2}\left(U_{k}\right)$ defined by

$$
h_{k}^{D}[v]=\left\|\left(\nabla-i \widetilde{\boldsymbol{a}_{k}}\right) v\right\|_{U_{k}}^{2}
$$

for $v \in Q\left(h_{k}^{D}\right):=W_{0, \widetilde{a_{k}}, 0}^{1}\left(U_{k}\right)$. From (2.10), (2.11) and (2.12), we obtain

$$
\begin{aligned}
h_{\Omega}^{R}[u] \geq & \left(h_{\Omega}^{D}-\epsilon_{R}\right)\left[\alpha_{1} u\right]+\left(h_{\Omega \cap(\partial \Omega)_{R}}^{D}-C_{1}-\epsilon_{R}\right)\left[\gamma_{0} u\right] \\
& +\sum_{k=1}^{K}\left(\frac{1}{2 C_{3}} h_{k}^{D}-C_{1}-2 C_{2}^{2}\left(\sigma^{-}\right)^{2}-\epsilon_{R}\right)\left[\frac{1}{\sqrt{2}}\left(\widetilde{\gamma_{k} u}\right)\right] .
\end{aligned}
$$

Next, define a map $j$ from $L^{2}(\Omega)$ to $L^{2}(\Omega) \oplus L^{2}\left(\Omega \cap(\partial \Omega)_{R}\right) \oplus\left(\oplus_{k=1}^{K} L^{2}\left(U_{k}\right)\right)$ by

$$
j v:=\left(\alpha_{1} v \oplus \gamma_{0} v \oplus\left(\oplus_{k=1}^{K}\left\{\frac{1}{\sqrt{2}} \widetilde{\gamma_{k} v}\right\}\right)\right)
$$

for $v \in L^{2}(\Omega)$. Since $\sum \alpha_{j}^{2}=\sum \beta_{k}^{2}=1$, we have by (2.11) that $j$ is an isometry. Moreover, since $j\left(Q\left(h_{\Omega}^{R}\right)\right) \subset Q\left(h_{\Omega}^{D}\right) \oplus Q\left(h_{\Omega \cap(\partial \Omega)_{R}}^{D}\right) \oplus\left(\oplus_{k=1}^{K} Q\left(h_{k}^{D}\right)\right)$, we have by (2.13) and [Cv, Lemme 5.1]

$$
\begin{aligned}
N_{\Omega}^{R}(\lambda) \leq & N_{\Omega}^{D}\left(\lambda+\epsilon_{R}\right)+N_{\Omega \cap(\partial \Omega)_{R}}^{D}\left(\lambda+C_{1}+\epsilon_{R}\right) \\
& +\sum_{k=1}^{K} N\left(2 C_{3}\left(\lambda+C_{1}+2 C_{2}^{2}\left(\sigma^{-}\right)^{2}+\epsilon_{R}\right) \mid h_{k}^{D}\right)
\end{aligned}
$$

for every $\lambda \in \boldsymbol{R}$, where $N\left(\lambda \mid h_{k}^{D}\right)$ denotes the number of eigenvalues less than or equal to $\lambda$ of the self-adjoint operator associated with the quadratic form $h_{k}^{D}$. By [D-I-M, Proposition 6.1], we have

$$
N_{\Omega \cap(\partial \Omega)_{R}}^{D}\left(\lambda+C_{1}+\epsilon_{R}\right) \leq M_{3}\left(\lambda+C_{1}+\epsilon_{R}\right)_{+}^{d / 2}\left|\Omega \cap(\partial \Omega)_{R}\right|
$$

and

$$
\begin{aligned}
& \sum_{k=1}^{K} N\left(2 C_{3}\left(\lambda+C_{1}+2 C_{2}^{2}\left(\sigma^{-}\right)^{2}+\epsilon_{R}\right) \mid h_{k}^{D}\right) \\
& \quad \leq \sum_{k=1}^{K} M_{3}\left(2 C_{3}\right)^{d / 2}\left(\lambda+C_{1}+2 C_{2}^{2}\left(\sigma^{-}\right)^{2}+\epsilon_{R}\right)_{+}^{d / 2}\left|U_{k}\right| \\
& \quad \leq B M_{3}\left(2 C_{3}\right)^{d / 2}\left(\lambda+C_{1}+2 C_{2}^{2}\left(\sigma^{-}\right)^{2}+\epsilon_{R}\right)_{+}^{d / 2}\left|(\partial \Omega)_{r}\right|,
\end{aligned}
$$

where we used (i) of [D-I-M, Proposition 4.1] and (v) of Definition 1.6. By (i) of [D-I-M, Proposition 4.5], there exists a constant $M_{4}>0$ dependent only on $d$,

$$
\left|(\partial \Omega)_{t}\right| \leq M_{4}(1+A) B \frac{t^{d}}{r^{d-1}}|\partial \Omega|^{\prime}
$$


for every $t \geq r$. By (2.14), (2.15), (2.16) and (2.17), we obtain

$$
\begin{aligned}
N_{\Omega}^{R}(\lambda) \leq & N_{\Omega}^{D}\left(\lambda+\epsilon_{R}\right)+C_{4} R^{d}\left(\lambda+C_{1}+\epsilon_{R}\right)_{+}^{d / 2}|\partial \Omega|^{\prime} \\
& +C_{5}\left(\lambda+C_{1}+2 C_{2}^{2}\left(\sigma^{-}\right)^{2}+\epsilon_{R}\right)_{+}^{d / 2}|\partial \Omega|^{\prime}
\end{aligned}
$$

for every $R \geq r$, where $C_{4}$ and $C_{5}$ is a positive constant dependent only on $r, A, B, d$.

Note that it is sufficient to show (1.5) for sufficiently small $\epsilon$. Take $\epsilon$ sufficiently small and put $R=\sqrt{2 M_{1}^{2} / \epsilon}$. Then we may assume $R \geq r$. By (2.18), we have

$$
N_{\Omega}^{R}(\lambda) \leq N_{\Omega}^{D}(\lambda+\epsilon)+C\left(\epsilon^{-d / 2}\left(\lambda+C_{0}\right)_{+}^{d / 2}+\left(\lambda+C_{0}+M_{0}\left(\sigma^{-}\right)^{2}\right)_{+}^{d / 2}\right)|\partial \Omega|^{\prime}
$$

for sufficiently small $\epsilon$, where $C=\max \left(C_{4}, C_{5}\right), C_{0}=C_{1}+1, M_{0}=2 C_{2}^{2}$. Therefore the assertion of the theorem holds.

\section{$\S 2.2$. Proof of Theorem 1.4}

We shall prove Theorem 1.4. When $\boldsymbol{a}=0$, we can get sharper estimate of $N_{U_{k}^{-}}^{R}(\lambda)$, by separation of variables.

Proposition 2.1. Let $r>0, A>0$ and $S \subset \boldsymbol{R}^{d-1}$ be a bounded open set. Let $\phi \in \operatorname{Lip}(S ; \boldsymbol{R})$ with $\|\nabla \phi\|_{\infty} \leq A$. Put

$$
\begin{aligned}
U & :=\left\{x=\left(x^{\prime}, x_{d}\right) \mid x^{\prime} \in S, \phi\left(x^{\prime}\right)-r<x_{d}<\phi\left(x^{\prime}\right)\right\}, \\
\Gamma: & =\left\{x=\left(x^{\prime}, x_{d}\right) \mid x^{\prime} \in S, x_{d}=\phi\left(x^{\prime}\right)\right\} .
\end{aligned}
$$

Let $\sigma \in L^{\infty}(\Gamma ; \boldsymbol{R})$. Define a sesqui-linear form $q_{U}^{R D}$ by

$$
q_{U}^{R D}(u, v)=(\nabla u, \nabla v)_{U}+\int_{\Gamma} \sigma \bar{u} v d S
$$

for $u, v \in Q\left(q_{U}^{R D}\right)$, where

$$
Q\left(q_{U}^{R D}\right):=\overline{\{u \in \operatorname{Lip}(\bar{U} ; \boldsymbol{C}) \mid \operatorname{supp} u \cap \partial U \subset \Gamma\}} .
$$

The overline denotes the closure with respect to the $W^{1}(U)$-norm. We denote the self-adjoint operator associated with the form $q_{U}^{R D}$ by $-\Delta_{U}^{R D}$ (the Laplacian with the Robin-Dirichlet mixed boundary conditions).

Then, there exist constants $M_{5}>1$ and $M_{6}>1$ dependent only on $A, d$ such that

$$
N\left(\lambda ;-\Delta^{R D}\right) \leq N\left(M_{5} \lambda ;-\Delta_{\widetilde{U}}^{D}\right)+N\left(M_{5} \lambda+\left(M_{6} \sigma^{-}\right)^{2} ;-\Delta_{S}^{D}\right)
$$


for every $\lambda \in \boldsymbol{R}$. Here, $N(\lambda ; H)$ is the eigenvalue counting function of a selfadjoint operator $H . \widetilde{U}:=S \times(0, r),-\Delta_{\widetilde{U}}^{D}$ is the Dirichlet Laplacian on $\widetilde{U}$ and $-\Delta_{S}^{D}$ is the Dirichlet Laplacian on the $(d-1)$-dimensional region $S . M_{6}=$ $\sqrt{1+A^{2}} M_{5} \cdot \sigma^{-}:=\max \left(\operatorname{ess} \cdot \sup (-\sigma), 1 /\left(M_{6} r\right)\right)$. below).

Remark. $\quad \operatorname{Lip}(\bar{U} ; \boldsymbol{C})$ is a dense subspace of $W^{1}(U)$ (see Proposition 3.1

Proof. Take a new coordinate $\xi=\left(\xi^{\prime}, \xi_{d}\right)$ defined on $\widetilde{U}$ by

$$
\left(x_{1}, \ldots, x_{d-1}, x_{d}\right)=\left(\xi_{1}, \ldots, \xi_{d-1}, \xi_{d}+\phi\left(\xi^{\prime}\right)-r\right)
$$

The transformation $\widetilde{U} \ni \xi \mapsto x \in U$ is a Lipschitz homeomorphism (see Definition 4.2) and the inverse is given by

$$
\left(\xi_{1}, \ldots, \xi_{d-1}, \xi_{d}\right)=\left(x_{1}, \ldots, x_{d-1}, x_{d}-\phi\left(x^{\prime}\right)+r\right) .
$$

Then, we have

$$
\begin{aligned}
\left(\partial_{\xi_{1}} u, \ldots, \partial_{\xi_{d-1}} u, \partial_{\xi_{d}} u\right) & =\left(\partial_{x_{1}} u+\partial_{x_{1}} \phi \partial_{x_{d}} u, \ldots, \partial_{x_{d-1}} u+\partial_{x_{d-1}} \phi \partial_{x_{d}} u, \partial_{x_{d}} u\right), \\
\left(\partial_{x_{1}} u, \ldots, \partial_{x_{d-1}} u, \partial_{x_{d}} u\right) & =\left(\partial_{\xi_{1}} u-\partial_{\xi_{1}} \phi \partial_{\xi_{d}} u, \ldots, \partial_{\xi_{d-1}} u-\partial_{\xi_{d-1}} \phi \partial_{\xi_{d}} u, \partial_{\xi_{d}} u\right)
\end{aligned}
$$

(note that the chain rule holds for the Lipschitz homeomorphisms; see Proposition 4.7 below). Since $\|\nabla \phi\|_{\infty} \leq A$, we have that the condition $u(x) \in W^{1}(U)$ is equivalent to the condition $u(\xi) \in W^{1}(\widetilde{U})$ (we abbreviate the function $x \mapsto u(x)$ as $u(x)$ and so on) and

$$
M_{5}^{-1}\left\|\nabla_{\xi} u\right\|_{\widetilde{U}}^{2} \leq\left\|\nabla_{x} u\right\|_{U}^{2} \leq M_{5}\left\|\nabla_{\xi} u\right\|_{\widetilde{U}}^{2}
$$

for $u \in W^{1}(\widetilde{U})$, where $M_{5}>1$ is a constant dependent only on $A, d$. Moreover,

$$
\begin{aligned}
\int_{\Gamma} \sigma\left(x^{\prime}\right)\left|u\left(x^{\prime}\right)\right|^{2} d S & =\int_{\widetilde{\Gamma}} \sigma\left(\xi^{\prime}\right)\left|u\left(\xi^{\prime}\right)\right|^{2} \sqrt{1+\left|\nabla \phi\left(\xi^{\prime}\right)\right|^{2}} d \xi^{\prime} \\
& \geq-\sqrt{1+A^{2}} \int_{\widetilde{\Gamma}} \sigma^{-}\left|u\left(\xi^{\prime}\right)\right|^{2} d \xi^{\prime}
\end{aligned}
$$

where $\widetilde{\Gamma}=S \times\{r\}, \sigma^{-}=\max \left(\operatorname{ess} \cdot \sup (-\sigma), 1 /\left(M_{6} r\right)\right)$ and $M_{6}=\sqrt{1+A^{2}} M_{5}$. We identify the surface measure on $\widetilde{\Gamma}$ with the Lebesgue measure $d \xi^{\prime}$ on $S$. Define a quadratic form $q_{\widetilde{U}}^{R D}$ on $L^{2}(\widetilde{U})$ by

$$
q_{\widetilde{U}}^{R D}[u]=\left\|\nabla_{\xi} u\right\|_{\widetilde{U}}^{2}-M_{6} \int_{\widetilde{\Gamma}} \sigma^{-}|u|^{2} d \xi^{\prime}
$$


for $u \in Q\left(q_{\widetilde{U}}^{R D}\right)=\overline{\{u \in \operatorname{Lip}(\overline{\widetilde{U}} ; \boldsymbol{C}) \mid \operatorname{supp} u \cap \partial \widetilde{U} \subset \widetilde{\Gamma}\}}$. Denote the self-adjoint operator associated with $q_{\widetilde{U}}^{R D}$ by $-\Delta_{\widetilde{U}}^{R D}$. Then we have by (2.20) and (2.21)

$$
q_{U}^{R D}[u] \geq M_{5}^{-1} q_{\widetilde{U}}^{R D}[u]
$$

Since the Jacobian $\operatorname{det}(\partial x / \partial \xi)=1$, the map $L^{2}(U) \ni u(x) \mapsto u(\xi) \in L^{2}(\widetilde{U})$ is an isometry. Thus, we obtain by the min-max principle

$$
N\left(\lambda ;-\Delta_{U}^{R D}\right) \leq N\left(M_{5} \lambda ;-\Delta_{\widetilde{U}}^{R D}\right) .
$$

Next, we shall calculate $N\left(\lambda ;-\Delta_{\widetilde{U}}^{R D}\right)$. Put $\nu=M_{6} \sigma^{-}$. Notice that $\nu$ is a positive constant. Since the region $\widetilde{U}$ is a product set $S \times(0, r)$, we can calculate the eigenvalues by separation of variables.

The operator domain of $-\Delta_{\widetilde{U}}^{R D}$ is

$D\left(-\Delta_{\widetilde{U}}^{R D}\right)=\left\{u \in W^{2}(\widetilde{U}) \mid \partial_{\xi_{d}} u(\xi)=\nu u(\xi)\right.$ for $\xi \in \widetilde{\Gamma}, u(\xi)=0$ for $\left.\xi \in \partial \widetilde{U} \backslash \widetilde{\Gamma}\right\}$.

The normalized eigenfunctions of $-\Delta_{\widetilde{U}}^{R D}$ are $\left\{\psi_{j}^{D}\left(\xi^{\prime}\right) \eta_{0}^{R D}\left(\xi_{d}\right)\right\}_{j=1}^{\infty} \cup\left\{\psi_{j}^{D}\left(\xi^{\prime}\right)\right.$ $\left.\times \eta_{k}^{R D}\left(\xi_{d}\right)\right\}_{j, k=1}^{\infty}$. Here, $\psi_{j}^{D}(j=1,2, \cdots)$ is the normalized eigenfunction corresponding to the $j$-th eigenvalue $\lambda_{j}^{D}$ of $-\Delta_{S}^{D} . \eta_{k}^{R D}(k=0,1,2, \cdots)$ is the normalized eigenfunction of $-\partial_{\xi_{d}}^{2}$ on $(0, r)$ with the boundary conditions

$$
\eta_{k}^{R D}(0)=0, \quad \partial_{\xi_{d}} \eta_{k}^{R D}(r)=\nu \eta_{k}^{R D}(r) .
$$

Notice that $r \geq 1 / \nu$, by the definition $\nu=M_{6} \sigma^{-}$. When $r>1 / \nu$, the function $\eta_{0}^{R D}\left(\xi_{d}\right)=c_{0} \sinh \left(\sqrt{\mu_{0}^{R D}} \xi_{d}\right)$ ( $c_{0}$ is the normalizing constant) corresponds to the unique negative eigenvalue $-\mu_{0}^{R D}$. In this case, (2.23) is equivalent to the condition

$$
\tanh \left(\sqrt{\mu_{0}^{R D}} r\right)=\frac{\sqrt{\mu_{0}^{R D}}}{\nu} .
$$

Examining the graph of two curves $y=\tanh r x, y=x / \nu$ in $x y$-plane, we conclude that there is a unique positive solution $\mu_{0}^{R D}$ of (2.24) and

$$
\mu_{0}^{R D} \leq \nu^{2}
$$

When $r=1 / \nu, \eta_{0}^{R D}\left(\xi_{d}\right)=c_{0} \xi_{d}$ is the eigenfunction corresponding to the eigenvalue $\mu_{0}^{R D}=0$, which clearly satisfies (2.25). The function $\eta_{k}^{R D}\left(\xi_{d}\right)=$ $c_{k} \sin \left(\sqrt{\mu_{k}^{R D}} \xi_{d}\right)(k=1,2, \cdots) \quad\left(c_{k}\right.$ is the normalizing constant) corresponds to the $k$-th positive eigenvalue $\mu_{k}^{R D}$. In this case, $(2.23)$ is equivalent to the condition

$$
\tan \left(\sqrt{\mu_{k}^{R D}} r\right)=\frac{\sqrt{\mu_{k}^{R D}}}{\nu} .
$$


Examining the graph of two curves $y=\tan r x, y=x / \nu$ in $x y$-plane, we conclude that the $k$-th eigenvalue $\mu_{k}^{R D}$ satisfies $((k / r) \pi)^{2}<\mu_{k}^{R D}<(((k+1) / r) \pi)^{2}$. Since $((k / r) \pi)^{2}$ is the $k$-th Dirichlet eigenvalue $\mu_{k}^{D}$ of $-\partial_{\xi_{d}}^{2}$ on $(0, r)$, we have

$$
\mu_{k}^{D}<\mu_{k}^{R D}<\mu_{k+1}^{D}
$$

The eigenvalues of $-\Delta_{\widetilde{U}}^{R D}$ are $\left\{\lambda_{j}^{D}-\mu_{0}^{R D}\right\}_{j=1}^{\infty} \cup\left\{\lambda_{j}^{D}+\mu_{k}^{R D}\right\}_{j, k=1}^{\infty}$. Thus, we obtain

$$
\begin{aligned}
& N\left(\lambda ;-\Delta_{\widetilde{U}}^{R D}\right) \\
& \quad=\#\left\{j \mid \lambda_{j}^{D}-\mu_{0}^{R D} \leq \lambda\right\}+\#\left\{(j, k) \mid \lambda_{j}^{D}+\mu_{k}^{R D} \leq \lambda\right\} \\
& \quad \leq \#\left\{j \mid \lambda_{j}^{D} \leq \lambda+\nu^{2}\right\}+\#\left\{(j, k) \mid \lambda_{j}^{D}+\mu_{k}^{D} \leq \lambda\right\} \\
& \quad=N\left(\lambda+\nu^{2} ;-\Delta_{S}^{D}\right)+N\left(\lambda ;-\Delta_{\widetilde{U}}^{D}\right),
\end{aligned}
$$

where we used (2.25) and (2.27) from the second line to the third.

By $(2.22),(2.28)$ and $\nu=M_{6} \sigma^{-}$, we reach the conclusion.

Proof of Theorem 1.4. By the same argument in the beginning of the proof of Theorem 1.2, we have (2.8).

Notice that $U_{k}^{-}=U_{k} \cap \Omega$ has a coordinate $y=\chi_{k}^{-1}(x)\left(y \in U_{k}^{-}, x \in\right.$ $\chi_{k}\left(U_{k}^{-}\right)$) (see Definition 1.6). Since $\chi_{k}$ is a congruent transformation, we can identify $U_{k}^{-}$with $\chi_{k}\left(U_{k}^{-}\right)$and define the quadratic form $q_{U_{k}^{-}}^{R D}$ and the RobinDirichlet Laplacian $-\Delta_{U_{k}^{-}}^{R D}$ as in Proposition 2.1. Since $\operatorname{supp} \gamma_{k} u \cap \partial U_{k}^{-} \subset$ $\partial U_{k}^{-} \cap \partial \Omega$, we have $\gamma_{k} u \in Q\left(q_{U_{k}^{-}}^{R D}\right)$.

Define a map $j$ from $L^{2}(\Omega)$ to $L^{2}(\Omega) \oplus L^{2}\left(\Omega \cap(\partial \Omega)_{R}\right) \oplus\left(\oplus_{k=1}^{K} L^{2}\left(U_{k}^{-}\right)\right)$by

$$
j v:=\left(\alpha_{1} v \oplus \gamma_{0} v \oplus\left(\oplus_{k=1}^{K}\left\{\gamma_{k} v\right\}\right)\right)
$$

for $v \in L^{2}(\Omega)$. Then, $j$ is an isometry and $j\left(Q\left(h_{\Omega}^{R}\right)\right) \subset Q\left(h_{\Omega}^{D}\right) \oplus Q\left(h_{\Omega \cap(\partial \Omega)_{R}}^{D}\right) \oplus$ $\left(\oplus_{k=1}^{K} Q\left(q_{U_{k}^{-}}^{R D}\right)\right)$. Thus, we have by $(2.8)$ and $[\mathrm{Cv}$, Lemme 5.1]

$$
\begin{aligned}
N_{\Omega}^{R}(\lambda) \leq & N_{\Omega}^{D}\left(\lambda+\epsilon_{R}\right)+N_{\Omega \cap(\partial \Omega)_{R}}^{D}\left(\lambda+C_{1}+\epsilon_{R}\right) \\
& +\sum_{k=1}^{K} N\left(\lambda+C_{1}+\epsilon_{R} ;-\Delta_{U_{k}^{-}}^{R D}\right)
\end{aligned}
$$

where we used $h_{U_{k}^{-}}^{R}\left[\gamma_{k} u\right] \geq q_{k}^{R D}\left[\gamma_{k} u\right]$ (remind $V \geq 0$ ). By Proposition 2.1 and 
[D-I-M, Proposition 6.1], we obtain

$$
\begin{array}{rl}
\sum_{k=1}^{K} & N\left(\lambda+C_{1}+\epsilon_{R} ;-\Delta_{U_{k}^{-}}^{R D}\right) \\
\leq & \sum_{k=1}^{K}\left\{N\left(M_{5}\left(\lambda+C_{1}+\epsilon_{R}\right) ;-\Delta \frac{D}{U_{k}^{-}}\right)\right. \\
& \left.+N\left(M_{5}\left(\lambda+C_{1}+\epsilon_{R}\right)+\left(M_{6} \sigma^{-}\right)^{2} ;-\Delta_{S_{k}(r)}^{D}\right)\right\} \\
\leq & \sum_{k=1}^{K}\left\{M_{3}\left(M_{5}\left(\lambda+C_{1}+\epsilon_{R}\right)\right)_{+}^{d / 2} \widetilde{U_{k}^{-} \mid}\right. \\
& \left.+M_{3}^{\prime}\left(M_{5}\left(\lambda+C_{1}+\epsilon_{R}\right)+\left(M_{6} \sigma^{-}\right)^{2}\right)_{+}^{(d-1) / 2}\left|S_{k}(r)\right|^{\prime}\right\} \\
\leq & C\left\{\left(\lambda+C_{1}+\epsilon_{R}\right)_{+}^{d / 2}+\left(\lambda+C_{1}+\epsilon_{R}+M_{0}\left(\sigma^{-}\right)^{2}\right)_{+}^{(d-1) / 2}\right\}|\partial \Omega|^{\prime},
\end{array}
$$

where $C>0$ is a constant dependent only on $r, A, B, d, M_{0}=\sqrt{1+A^{2}} M_{6}>1$ and $\sigma^{-}=\max \left(\operatorname{ess} \cdot \sup (-\sigma), \sqrt{1+A^{2}} /\left(M_{0} r\right)\right)=\max \left(\operatorname{ess} \cdot \sup (-\sigma), 1 /\left(M_{6} r\right)\right)$. Here, we used (i) of [D-I-M, Proposition 4.1], (v) of Definition 1.6 and (2.17).

Put $R=\sqrt{2 M_{1} / \epsilon}$ for sufficiently small $\epsilon>0$. Then we obtain from (2.29), (2.30), (2.15) and (2.17),

$$
\begin{aligned}
N_{\Omega}^{R}(\lambda) \leq & N_{\Omega}^{D}\left(\lambda+\epsilon_{R}\right)+C R^{d}\left(\lambda+C_{1}+\epsilon_{R}\right)_{+}^{d / 2}|\partial \Omega|^{\prime} \\
& +C\left(\lambda+C_{1}+\epsilon_{R}+M_{0}\left(\sigma^{-}\right)^{2}\right)_{+}^{(d-1) / 2}|\partial \Omega|^{\prime} \\
\leq & N_{\Omega}^{D}(\lambda+\epsilon)+C \epsilon^{-d / 2}\left(\lambda+C_{0}\right)_{+}^{d / 2}|\partial \Omega|^{\prime} \\
& +C\left(\lambda+C_{0}+M_{0}\left(\sigma^{-}\right)^{2}\right)_{+}^{(d-1) / 2}|\partial \Omega|^{\prime},
\end{aligned}
$$

where $C>0$ is a constant dependent only on $r, A, B, d$ and $C_{0}=C_{1}+1$. Therefore the assertion holds.

\section{§3. Trace Estimate for Magnetic Sobolev Spaces}

In this section, we shall prove that the restriction operator $\left.\cdot\right|_{\partial \Omega}$ defined on $\operatorname{Lip}(\bar{\Omega})$ (the space of complex-valued Lipschitz continuous functions on $\bar{\Omega}$ ) can be extended to the space $W_{a, V}^{1}(\Omega)$. In the sequel, the symbol $K \subset \subset U$ means that $K$ is a compact set, $U$ is an open set and $K \subset U$.

Proposition 3.1. Let $\Omega \in L M(r, A, B)$ for some $r, A, B>0$. Then, $\operatorname{Lip}(\bar{\Omega})$ is dense in $W_{a, V}^{1}(\Omega)$. 
Proof. Let $\left(\Omega,\left\{U_{k}, \chi_{k}, S_{k}, \phi_{k}\right\}_{k=1}^{K}\right) \in L M(r, A, B)$ and take $u \in W_{a, V}^{1}(\Omega)$. Take a partition of unity $\left\{\beta_{k}\right\}_{k=0}^{K}$ appeared in the beginning of the proof of Theorem 1.2 and put $\gamma_{k}=\beta_{k}^{2}$ for $k=0,1, \ldots, K$. Since $\operatorname{supp} \gamma_{0} \subset \subset \Omega$, $\gamma_{0} u \subset W_{0, a, V}^{1}(\Omega) \subset W_{0, a, V}^{1}\left(\boldsymbol{R}^{d}\right)$ (see (v) of [D-I-M, Proposition 2.1]). Thus, we can approximate $\gamma_{0} u$ by $C_{0}^{\infty}(\Omega)$-functions with respect to $W_{a, V}^{1}(\Omega)$-norm (see [L-S]). Since $\operatorname{supp} \gamma_{k} u \subset U_{k}^{-}$and $\operatorname{supp} \gamma_{k} u \cap \partial U_{k}^{-} \subset \Gamma_{k}\left(\Gamma_{k}=\partial \Omega \cap U_{k}\right)$ for $k=1, \ldots, K$, we can construct the extended functions $\widetilde{\gamma_{k} u}, \widetilde{V_{k}}, \widetilde{\boldsymbol{a}_{k}}$ defined on $U_{k}$, which are the extension of the functions $\gamma_{k} u, \boldsymbol{a}, V$ defined on $U_{k}^{-}$respectively and satisfy $\widetilde{\gamma_{k} u} \in W_{0, \widetilde{a_{k}}, \widetilde{V_{k}}}^{1}\left(U_{k}\right)$ (see Section 5 in [D-I-M]). Thus, we can approximate $\widetilde{\gamma_{k} u}$ by $C_{0}^{\infty}\left(U_{k}\right)$-functions with respect to $W_{\widetilde{a_{k}}, \widetilde{V_{k}}}^{1}\left(U_{k}\right)$-norm. Taking the restriction of these functions, we can approximate $\gamma_{k} u$ by $\operatorname{Lip}\left(\overline{U_{k}^{-}}\right)$functions whose support touch the boundary of $\partial \Omega$ only on $\Gamma_{k}$, with respect to $W_{a, V}^{1}\left(U_{k}^{-}\right)$-norm. Since $u=\sum_{k=0}^{K} \gamma_{k} u$, we obtain the conclusion.

Note that, we can similarly prove that $\operatorname{Lip}(\bar{\Omega})$ is dense in the space $W^{1, p}(\Omega)$ $(1 \leq p<\infty)$, where

$$
W^{1, p}(\Omega)=\left\{u \in L^{p}(\Omega) \mid \nabla u \in\left(L^{p}(\Omega)\right)^{d}\right\} .
$$

These facts enable us to use the usual approximation arguments.

Proposition 3.2. Let $\Omega \in L M(r, A, B)$ for some $r, A, B>0$ and $1 \leq$ $p<\infty$. Then, we have the following:

(i) The restriction operator $\left.\cdot\right|_{\partial \Omega}$ defined on Lip $(\bar{\Omega})$ is extended to the bounded operator from $W^{1, p}(\Omega)$ to $L^{p}(\partial \Omega, d S)$. Moreover, there exists a constant $C_{p}=C_{p}(p, r, A, B, d)>0$ such that

$$
\|u\|_{L^{p}(\partial \Omega, d S)}^{p} \leq C_{p}\left(\epsilon^{-1}\|u\|_{L^{p}(\Omega)}^{p}+\epsilon^{p-1}\|\nabla u\|_{\left(L^{p}(\Omega)\right)^{d}}^{p}\right)
$$

for every $u \in W^{1, p}(\Omega)$ and $0<\epsilon<r$.

(ii) For any $\boldsymbol{a} \in\left(L^{2}(\Omega ; \boldsymbol{R})\right)^{d}$ and $V \in L^{1}(\Omega ; \boldsymbol{R})$ with $V \geq 0$, the restriction operator $\left.\right|_{\partial \Omega}$ defined on $\operatorname{Lip}(\bar{\Omega})$ is extended to the bounded operator from $W_{a, V}^{1}(\Omega)$ to $L^{2}(\partial \Omega, d S)$. Moreover, there exists a constant $C_{2}=$ $C_{2}(r, A, B, d)>0$ (independent of $\left.\boldsymbol{a}, V\right)$ such that

$$
\|u\|_{L^{2}(\partial \Omega, d S)}^{2} \leq C_{2}\left(\epsilon^{-1}\|u\|_{L^{2}(\Omega)}^{2}+\epsilon\|(\nabla-i \boldsymbol{a}) u\|_{\left(L^{2}(\Omega)\right)^{d}}^{2}\right)
$$

for every $u \in W_{a, V}^{1}(\Omega)$ and $0<\epsilon<r$. 
Proof. (i) By the remark after Proposition 3.1, we see that it is sufficient to show (3.2) for $u \in \operatorname{Lip}(\bar{\Omega})$.

Take $\left(\Omega,\left\{U_{k}, \chi_{k}, S_{k}, \phi_{k}\right\}_{k=1}^{K}\right) \in L M(r, A, B)$. Put $\Gamma_{k}=U_{k} \cap \partial \Omega$ and $U_{k}^{-}=U_{k} \cap \Omega$. First, we show that there exists a constant $C=C(p, r, A, d)>0$ such that

$$
\|u\|_{L^{p}\left(\Gamma_{k}, d S\right)}^{p} \leq C\left(\epsilon^{-1}\|u\|_{L^{p}\left(U_{k}^{-}\right)}^{p}+\epsilon^{p-1}\|\nabla u\|_{\left(L^{p}\left(U_{k}^{-}\right)\right)^{d}}^{p}\right)
$$

for every $u \in \operatorname{Lip}\left(\overline{U_{k}^{-}}\right)$with $\operatorname{supp} u \cap \partial U_{k}^{-} \subset \Gamma_{k}$. By (ii) of Definition 1.6,

$$
\chi_{k}\left(U_{k}^{-}\right)=\left\{\left(x^{\prime}, x_{d}\right) \in \boldsymbol{R}^{d} \mid x^{\prime} \in S_{k}(r), \phi_{k}\left(x^{\prime}\right)-r<x_{d}<\phi_{k}\left(x^{\prime}\right)\right\} .
$$

Since $\chi_{k}$ is a congruent transformation, we may assume $\chi_{k}$ is an identity map without loss of generality.

For $r>\epsilon>0$, take $\eta_{\epsilon}=\eta_{\epsilon}\left(x_{d}\right) \in C^{\infty}((-r, 0))$ such that $0 \leq \eta_{\epsilon}\left(x_{d}\right) \leq 1$,

$$
\eta_{\epsilon}\left(x_{d}\right)= \begin{cases}1 & \text { if } \quad-\epsilon / 2 \leq x_{d}<0 \\ 0 & \text { if } \quad x_{d}<-\epsilon\end{cases}
$$

and

$$
\left|\partial_{x_{d}} \eta_{\epsilon}\right| \leq C_{0} \epsilon^{-1}
$$

for some constant $C_{0}>0$ independent of $\epsilon$.

Since $u$ is Lipschitz continuous, $u$ is absolutely continuous and $\partial_{x_{d}} u \in L^{\infty}$. So we have

$$
u\left(x^{\prime}, \phi\left(x^{\prime}\right)\right):=\int_{-\epsilon}^{0} \partial_{x_{d}}\left(\eta_{\epsilon}\left(x_{d}\right) u\left(x^{\prime}, \phi\left(x^{\prime}\right)+x_{d}\right)\right) d x_{d},
$$

where we used $\eta_{\epsilon}(0)=1$ and $\eta_{\epsilon}(-\epsilon)=0$. Moreover,

$$
\begin{aligned}
\int_{\Gamma_{k}} \mid & \left.u\left(\phi\left(x^{\prime}\right)\right)\right|^{p} d S \\
\leq & \int_{S_{k}(r)} \sqrt{1+\left|\nabla \phi\left(x^{\prime}\right)\right|^{2}} d x^{\prime} \\
& \times\left(\int_{-\epsilon}^{0}\left|\left(\partial_{x_{d}} \eta_{\epsilon}\right)\left(x_{d}\right) u\left(x^{\prime}, \phi\left(x^{\prime}\right)+x_{d}\right)+\eta_{\epsilon}\left(x_{d}\right)\left(\partial_{x_{d}} u\right)\left(x^{\prime}, \phi_{k}\left(x^{\prime}\right)+x_{d}\right)\right| d x_{d}\right)^{p} \\
\leq & 2^{p-1} \sqrt{1+A^{2}} \int_{S_{k}(r)} \epsilon^{p-1} d x^{\prime} \\
& \times\left(C_{0}^{p} \epsilon^{-p} \int_{-\epsilon}^{0}\left|u\left(x^{\prime}, \phi\left(x^{\prime}\right)+x_{d}\right)\right|^{p} d x_{d}+\int_{-\epsilon}^{0}\left|\partial_{x_{d}} u\left(x^{\prime}, \phi\left(x^{\prime}\right)+x_{d}\right)\right|^{p} d x_{d}\right) \\
\leq & C\left(\epsilon^{-1}\|u\|_{L^{p}\left(U_{k}^{-}\right)}^{p}+\epsilon^{p-1}\left\|\partial_{x_{d}} u\right\|_{L^{p}\left(U_{k}^{-}\right)}^{p}\right)
\end{aligned}
$$


where we used the Hölder inequality, $\|\nabla \phi\|_{\infty} \leq A$, (3.5) and the inequality $(s+t)^{p} \leq 2^{p-1}\left(s^{p}+t^{p}\right)$ for $s, t \geq 0$, in the second inequality. Thus we have (3.4).

Next, Take $u \in \operatorname{Lip}(\bar{\Omega})$ and a partition of unity $\left\{\beta_{k}\right\}_{k=0}^{K}$ appeared in the beginning of the proof of Theorem 1.2 and put $\gamma_{k}=\beta_{k}^{2}(k=0,1, \ldots, K)$. Then $u=\sum_{k=0}^{K} \gamma_{k} u$, supp $\gamma_{0} \subset \subset \Omega$ and supp $\gamma_{k} u \cap \partial \Omega \subset \Gamma_{k}$. Thus $\left.u\right|_{\partial \Omega}=$ $\left.\sum_{k=1}^{K} \gamma_{k} u\right|_{\Gamma_{k}}$. By (v) of Definition 1.6, we see that there exists a constant $C=C(p, r, A, B, d)>0$ such that

$$
C^{-1} \leq \sum_{k=1}^{K}\left|\gamma_{k}(x)\right|^{p} \leq C, \quad \sum_{k=1}^{K}\left|\nabla \gamma_{k}(x)\right|^{p} \leq C
$$

for every $x \in \bar{\Omega}$, where we used $\sum \gamma_{k}=1$. Hence we have

$$
\begin{aligned}
& \|u\|_{L^{p}(\partial \Omega, d S)}^{p} \\
& \quad \leq C \sum_{k=1}^{K}\left\|\gamma_{k} u\right\|_{L^{p}\left(\Gamma_{k}, d S\right)}^{p} \\
& \quad \leq C \sum_{k=1}^{K}\left(\epsilon^{-1}\left\|\gamma_{k} u\right\|_{L^{p}(\Omega)}^{p}+\epsilon^{p-1}\left\|\left(\nabla \gamma_{k}\right) u\right\|_{\left(L^{p}(\Omega)\right)^{d}}^{p}+\epsilon^{p-1}\left\|\gamma_{k}(\nabla u)\right\|_{\left(L^{p}(\Omega)\right)^{d}}^{p}\right) \\
& \quad \leq C\left(\left(\epsilon^{-1}+\epsilon^{p-1}\right)\|u\|_{L^{p}(\Omega)}^{p}+\epsilon^{p-1}\|\nabla u\|_{\left(L^{p}(\Omega)\right)^{d}}^{p}\right) \\
& \quad \leq C\left(\epsilon^{-1}\|u\|_{L^{p}(\Omega)}^{p}+\epsilon^{p-1}\|\nabla u\|_{\left(L^{p}(\Omega)\right)^{d}}^{p}\right),
\end{aligned}
$$

for $r>\epsilon>0$. Here, we write every constant dependent only on $p, r, A, B, d$ by the same letter $C$. We used (3.4) in the second inequality.

(ii) By Proposition 3.1, we see that it is sufficient to show (3.3) for $u \in$ $\operatorname{Lip}(\bar{\Omega})$.

Take $u \in \operatorname{Lip}(\bar{\Omega})$. Then, $|u| \in \operatorname{Lip}(\bar{\Omega}) \subset W^{1,1}(\Omega)$ and $\partial_{j}|u|=\operatorname{Re}((\operatorname{sgn} \bar{u})$ $\times \partial_{j} u$ ) for $1 \leq j \leq m$, where

$$
\operatorname{sgn} z=\left\{\begin{array}{cc}
\frac{z}{|z|} & (z \neq 0) \\
0 & (z=0)
\end{array}\right.
$$

(see [D-I-M, Corollary 2.3]). Thus, $\partial_{j}|u|=\operatorname{Re}\left((\operatorname{sgn} \bar{u})\left(\partial_{j}-i a_{j}\right) u\right)$ and $\left|\partial_{j}\right| u|| \leq$ $\left|\left(\partial_{j}-i a_{j}\right) u\right|$. Hence

$$
\|\nabla|u|\|_{\left(L^{2}(\Omega)\right)^{d}}^{2} \leq\|(\nabla-i \boldsymbol{a}) u\|_{\left(L^{2}(\Omega)\right)^{d}}^{2} .
$$


By (3.6) and (3.2) for $p=2$, we obtain

$$
\begin{aligned}
\|u\|_{L^{2}(\partial \Omega, d S)}^{2} & =\||u|\|_{L^{2}(\partial \Omega, d S)}^{2} \\
& \leq C_{2}\left(\epsilon^{-1}\|u\|_{L^{2}(\Omega)}^{2}+\epsilon\|\nabla|u|\|_{\left(L^{2}(\Omega)\right)^{d}}^{2}\right) \\
& \leq C_{2}\left(\epsilon^{-1}\|u\|_{L^{2}(\Omega)}^{2}+\epsilon\|(\nabla-i \boldsymbol{a}) u\|_{\left(L^{2}(\Omega)\right)^{d}}^{2}\right)
\end{aligned}
$$

for $r>\epsilon>0$. Thus, we obtain the conclusion.

\section{$\S 4$. Fundamental Formulas for Lipschitz Domains}

In the present paper and the previous paper [D-I-M], we used several fundamental formulas for Lipschitz domains, e.g. the chain rule, the change of variables formula, the divergence formula. But one may doubt whether such formulas for Lipschitz domains holds. For example, the gradient matrix $\nabla T$ of a Lipschitz continuous transformation $T$ or the unit outer normal vector $\boldsymbol{n}$ on the boundary of a Lipschitz domain is generally discontinuous. For this reason, Wloka avoids using the change of variables formula for Lipschitz continuous transformations in his book (see [Wl, p. 84]). Unfortunately, the author cannot find a rigorous proof of such formulas in recent texts (the change of variables formula for Lipschitz domains was proved by Rademacher [Ra]; his paper was written in 1919). We shall give a proof of such formulas for readers' use and for the sake of completeness.

\section{§4.1. Total differentiability of Lipschitz continuous functions}

In this subsection, we shall remind an old result about the total differentiability of Lipschitz continuous functions.

Definition 4.1. Let $U$ be an open set in $\boldsymbol{R}^{n}$. We say a map $T: U \rightarrow$ $\boldsymbol{R}^{m}$ is totally differentiable at $x \in U$ if there exists a linear map $\nabla T(x): \boldsymbol{R}^{n} \rightarrow$ $\boldsymbol{R}^{m}$ such that

$$
\limsup _{h \in \mathbf{R}^{n},|h| \searrow 0} \frac{1}{|h|}|T(x+h)-T(x)-\nabla T(x) \cdot h|=0 .
$$

Rademacher [Ra] proves the following:

Theorem 4.1 (Rademacher). Suppose $f$ be a continuous function defined on an open set $D \subset \boldsymbol{R}^{2}$ and satisfies

$$
L(x, y):=\limsup _{|h|^{2}+|k|^{2} \searrow 0} \frac{|f(x+h, y+k)-f(x, y)|}{\sqrt{h^{2}+k^{2}}}<\infty, \quad \text { a.e. }(x, y) \in D .
$$

Then, $f(x, y)$ is totally differentiable at almost every $(x, y) \in D$. 
This immediately implies the total differentiability of Lipschitz continuous functions on a domain in $\boldsymbol{R}^{2}$. Moreover, the proof of Rademacher's theorem can be applied to the multi-dimensional case with a little modification. Hence the following holds:

Corollary 4.2. Let $U$ be an open set in $\boldsymbol{R}^{n}$ and $T: U \rightarrow \boldsymbol{R}^{m}$ be a Lipschitz continuous map. Then, for almost every $x \in U, T$ is totally differentiable at $x$ and the matrix $\nabla T(x)$ is given by

$$
\nabla T(x)=\left(\frac{\partial T}{\partial x_{1}}(x), \ldots, \frac{\partial T}{\partial x_{n}}(x)\right),
$$

where $\partial T / \partial x_{j}(x)$ is the column vector ${ }^{t}\left(\left(\partial T_{1} / \partial x_{j}\right)(x), \ldots,\left(\partial T_{m} / \partial x_{j}\right)(x)\right)$ for $j=1, \ldots, n$.

In the present paper, we need only Corollary 4.2. For readers' use, we shall give an elementary proof of Corollary 4.2 , in which we do not use Rademacher's theorem but the following well-known result:

Proposition 4.3. Let $U$ be an open set in $\boldsymbol{R}^{n}$ and $T: U \rightarrow \boldsymbol{R}^{m}$ a Lipschitz continuous map. Then, for every $\omega \in S^{n-1}$, the directional derivative

$$
\nabla_{\omega} T(x):=\lim _{t \searrow 0} \frac{1}{t}(T(x+\omega t)-T(x))
$$

exists at almost every $x \in U$ and $\nabla_{\omega} T$ is $L^{\infty}(U)$-valued vector. Moreover, when $\omega=\mathbf{e}_{j}:={ }^{t}\left(\delta_{1 j}, \ldots, \delta_{n j}\right)$ ( $\delta$ is the Kronecker's delta), the function $\nabla_{\mathbf{e}_{j}} T$ agrees with the distributional derivative $\partial T / \partial x_{j}$.

This proposition follows from the fact that a Lipschitz continuous function on the real axis is absolutely continuous (see e.g. [Wl, Theorem 1.8]).

Proof of Corollary 4.2. Let $U$ and $T$ satisfy the assumption. Put $C_{1}=$ $\sup _{x \neq y}(|T(x)-T(y)| /|x-y|)<\infty$. We shall divide the proof into three steps. In the sequel, we say a measurable set $S \subset U$ is a full-measure set if $|U \backslash S|=0$.

Step 1. For almost every $x \in U$, the directional derivative $\nabla_{\omega} T(x)$ exists for every $\omega \in S^{n-1}$ and

$$
\lim _{t \downarrow 0}\left(\sup _{\omega \in S^{n-1}} \frac{1}{t}\left|T(x+\omega t)-T(x)-\nabla_{\omega} T(x) t\right|\right)=0 .
$$

Moreover, the map $S^{n-1} \ni \omega \rightarrow \nabla_{\omega} T(x) \in \boldsymbol{R}^{m}$ is continuous. 
Proof of Step 1. Let $\left\{\omega_{j}\right\}_{j=1}^{\infty}$ be a dense countable set in $S^{n-1}$. By Proposition 4.3,

$$
U_{j}:=\left\{x \in U \mid \nabla_{\omega_{j}} T(x) \text { exists. }\right\}
$$

is a full-measure set for each $j \geq 1$. Put $U_{0}:=\cap_{j=1}^{\infty} U_{j}$. Then $U_{0}$ is a fullmeasure set.

Fix $x \in U_{0}$. Then, we have

$$
\left|\frac{T\left(x+\omega_{j} t\right)-T(x)}{t}-\frac{T\left(x+\omega_{k} t\right)-T(x)}{t}\right| \leq C_{1}\left|\omega_{j}-\omega_{k}\right|
$$

for every $j, k \geq 1$ and sufficiently small $t>0$. Taking the limit as $t \downarrow 0$, we have

$$
\left|\nabla_{\omega_{j}} T(x)-\nabla_{\omega_{k}} T(x)\right| \leq C_{1}\left|\omega_{j}-\omega_{k}\right| .
$$

By (4.2), there exists a continuous function $F_{x}(\omega)$ of $\omega \in S^{n-1}$ such that

$$
F_{x}\left(\omega_{j}\right)=\nabla_{\omega_{j}} T(x)
$$

for every $j \geq 1$ and

$$
\left|F_{x}(\omega)-F_{x}\left(\omega^{\prime}\right)\right| \leq C_{1}\left|\omega-\omega^{\prime}\right|
$$

for every $\omega, \omega^{\prime} \in S^{n-1}$.

Take $\epsilon>0$. Since $x \in U_{j}$, there exists a number $t_{j}>0$ such that

$$
\left|\frac{T\left(x+\omega_{j} t\right)-T(x)}{t}-F_{x}\left(\omega_{j}\right)\right|<\frac{\epsilon}{3}
$$

for $0<t<t_{j}$. Put

$$
W_{j}:=\left\{\omega \in S^{n-1}|| \omega-\omega_{j} \mid<\epsilon /\left(3 C_{1}\right)\right\} .
$$

For $\omega \in W_{j}$, we have

$$
\begin{aligned}
& \left|\frac{T(x+\omega t)-T(x)}{t}-F_{x}(\omega)\right| \\
& \leq\left|\frac{T(x+\omega t)-T(x)}{t}-\frac{T\left(x+\omega_{j} t\right)-T(x)}{t}\right| \\
& \quad+\left|\frac{T\left(x+\omega_{j} t\right)-T(x)}{t}-F_{x}\left(\omega_{j}\right)\right|+\left|F_{x}\left(\omega_{j}\right)-F_{x}(\omega)\right|
\end{aligned}
$$

for $0<t<t_{j}$, where we used (4.1), (4.4) and (4.5). 
Since $S^{n-1}=\cup_{j=1}^{\infty} W_{j}$ (notice that $\left\{\omega_{j}\right\}_{j=1}^{\infty}$ is dense in $S^{n-1}$ and that the number $\epsilon /\left(3 C_{1}\right)$ is independent of $\left.j\right)$ and $S^{n-1}$ is compact, there exists a finite number of indices $\left\{j_{1}, \ldots, j_{N}\right\}$ such that $S^{n-1}=\cup_{k=1}^{N} W_{j_{k}}$. Put $t_{0}=$ $\min _{k=1, \ldots, N} t_{j_{k}}$. Then, we have

$$
\left|\frac{T(x+\omega t)-T(x)}{t}-F_{x}(\omega)\right|<\epsilon
$$

for every $\omega \in S^{n-1}$ and $0<t<t_{0}$. Thus we obtain the conclusion since $x \in U_{0}$ is arbitrary and $U_{0}$ is a full-measure set.

Step 2. Put $\nabla T(x):=\left(\partial T / \partial x_{1}, \ldots, \partial T / \partial x_{n}\right)$. Then,

$$
\lim _{t \downarrow 0}\left(\sup _{\omega \in S^{n-1}} \int_{K}\left|\frac{T(x+\omega t)-T(x)}{t}-\nabla T(x) \cdot \omega\right| d x\right)=0
$$

for every compact set $K \subset U$.

Proof of Step 2. For $\omega={ }^{t}\left(\omega_{1}, \ldots, \omega_{n}\right)$, put $\omega^{(j)}={ }^{t}\left(\omega_{1}, \ldots, \omega_{j}, 0, \ldots, 0\right)$ and $\omega^{(0)}={ }^{t}(0, \ldots, 0)$. Then we have

$$
\begin{aligned}
& \frac{T(x+\omega t)-T(x)}{t}-\nabla T(x) \cdot \omega \\
& \quad=\sum_{j=1}^{n} \omega_{j}\left\{\int_{0}^{1}\left(\frac{\partial T}{\partial x_{j}}\left(x+\omega^{(j-1)} t+s \omega_{j} t \boldsymbol{e}_{j}\right)-\frac{\partial T}{\partial x_{j}}(x)\right) d s\right\} .
\end{aligned}
$$

Hence we have

$$
\begin{aligned}
& \int_{K}\left|\frac{T(x+\omega t)-(x)}{t}-\nabla T(x) \cdot \omega\right| d x \\
& \leq \sum_{j=1}^{n} \sup _{|y| \leq t} \int_{K}\left|\frac{\partial T}{\partial x_{j}}(x+y)-\frac{\partial T}{\partial x_{j}}(x)\right| d x .
\end{aligned}
$$

Since $\partial T / \partial x_{j} \in L^{\infty}(U)$, the map $y \mapsto \partial T /\left.\partial x_{j}(\cdot-y)\right|_{K} \in L^{1}(K)$ is continuous (see [Ru1, Theorem 9.5]). Thus the right hand side of (4.8) goes to 0 as $t \downarrow 0$ and is independent of $\omega \in S^{n-1}$. Therefore we obtain the conclusion.

Step 3. For almost every $x \in U$,

$$
\nabla_{\omega} T(x)=\nabla T(x) \cdot \omega
$$

for every $\omega \in S^{n-1}$. 
Proof of Step 3. Let $\omega_{j}, U_{j}, U_{0}$ as in the proof of Step 1. By Step 2, the function $x \mapsto(T(x+\omega t)-T(x)) / t$ converges to $\nabla T(x) \cdot \omega$ as $t \downarrow 0$ in $L_{l o c}^{1}(U)$. Then, for $j \geq 1$, there exists a full-measure set $V_{j}$ and a sequence $\left\{t_{k}^{(j)}\right\}_{k=1}^{\infty}$ such that for every $x \in V_{j}$

$$
\frac{T\left(x+\omega_{j} t_{k}^{(j)}\right)-T(x)}{t_{k}^{(j)}} \rightarrow \nabla T(x) \cdot \omega_{j}
$$

as $k \rightarrow \infty$. By Step 1 , for every $x \in U_{0}$

$$
\frac{T\left(x+\omega_{j} t_{k}^{(j)}\right)-T(x)}{t_{k}^{(j)}} \rightarrow \nabla_{\omega_{j}} T(x)
$$

as $k \rightarrow \infty$. Put $V_{0}=\cap_{j=1}^{\infty} V_{j} \cap U_{0}$. Then, $V_{0}$ is a full-measure set and

$$
\nabla_{\omega_{j}} T(x)=\nabla T(x) \cdot \omega_{j}
$$

for every $x \in V_{0}, j \geq 1$. Since the map $S^{n-1} \ni \omega \mapsto \nabla_{\omega} T(x)$ is continuous for each $x \in V_{0}$ by Step 1 and $\left\{\omega_{j}\right\}_{j=1}^{\infty}$ is dense in $S^{n-1}$, we obtain the conclusion.

By the assertions of Steps 1 and 3, Corollary 4.2 holds.

\section{§4.2. Change of variables and Chain rule}

We see that the change of variables formula holds for Lipschitz continuous coordinate change.

Definition 4.2. Let $U$ and $V$ be open sets in $\boldsymbol{R}^{n}$. We say a map $T: U \rightarrow V$ is a Lipschitz homeomorphism if $T$ is bijective, Lipschitz continuous, and the inverse map $T^{-1}: V \rightarrow U$ is also Lipschitz continuous.

The following lemma is given in [Ru1, Theorem 7.26].

Lemma 4.4. Let $X$ be a Lebesgue measurable subset of $\boldsymbol{R}^{n}$ and $U$ be an open subset of $\boldsymbol{R}^{n}$ with $X \subset U \subset \boldsymbol{R}^{n}$. Suppose that $T: U \rightarrow \boldsymbol{R}^{n}$ is an injective continuous map and $T$ is totally differentiable at every point of $X$. Moreover, suppose that $|T(U \backslash X)|=0$. Then,

$$
\int_{T(X)} f(y) d y=\int_{X}(f \circ T)(x)|\operatorname{det} \nabla T(x)| d x
$$

for every nonnegative measurable function $f$ on $\boldsymbol{R}^{n}$.

Together with Corollary 4.2, this lemma proves the change of variables formula: 
Corollary 4.5. Let $U$ be an open set in $\boldsymbol{R}^{n}$ and $T$ be an injective Lipschitz continuous map from $U$ to $\boldsymbol{R}^{n}$. Let $f \in L^{1}(T(U))$. Then, $(f \circ$ $T)|\operatorname{det} \nabla T| \in L^{1}(U)$ and

$$
\int_{T(U)} f(y) d y=\int_{U} f \circ T(x)|\operatorname{det} \nabla T(x)| d x .
$$

Proof. Let $T$ be an injective Lipschitz continuous map. By Corollary 4.2, the map $T$ is totally differentiable on a set $U_{0}$ with $\left|U \backslash U_{0}\right|=0$. Put $X=U_{0}$. Then, all assumptions of Lemma 4.4 are satisfied, since a Lipschitz continuous map transfers a zero-measure set into a zero-measure set (see [Wl, Lemma 2.3]). Since $\left|U \backslash U_{0}\right|=0$, we have (4.11) for $f \geq 0$, which clearly implies the remaining assertions.

For a map $T: U \rightarrow V$, we define the pull-back operator $T^{*}$ by $T^{*} f=f \circ T$ for a measurable function $f$ on $V$. Then, the following holds clearly from Corollary 4.5:

Proposition 4.6. Let $U$ and $V$ be open sets in $\boldsymbol{R}^{n}$ and $T: U \rightarrow V$ is a Lipschitz homeomorphism. Then, for $1 \leq p<\infty$, the pull-back operator $T^{*}$ is an isomorphism (linear bijective, bicontinuous map) from $L^{p}(V)$ to $L^{p}(U)$ and

$$
\left\|T^{*}\right\|_{B\left(L^{p}(V), L^{p}(U)\right)} \leq\left\|\nabla\left(T^{-1}\right)\right\|_{\infty}^{n / p}, \quad\left\|\left(T^{-1}\right)^{*}\right\|_{B\left(L^{p}(U), L^{p}(V)\right)} \leq\|\nabla T\|_{\infty}^{n / p} .
$$

Moreover, if $|\operatorname{det} \nabla T(x)|=1$ for almost every $x \in U$, the operator $T^{*}$ is an isometric isomorphism from $L^{p}(V)$ to $L^{p}(U)$.

We shall prove the chain rule for coordinate change by Lipschitz homeomorphisms. Remind that $W^{1, p}(\Omega)$ is the Sobolev space defined by (3.1):

Proposition 4.7. Let $U$ and $V$ be open sets in $\boldsymbol{R}^{n}$ and $T: U \rightarrow V$ is a Lipschitz homeomorphism. Then, for $1 \leq p<\infty$ the pull-back operator $T^{*}$ is an isomorphism from $W^{1, p}(V)$ to $W^{1, p}(U)$ and

$$
\begin{aligned}
\left\|T^{*}\right\|_{B\left(W^{1, p}(V), W^{1, p}(U)\right)} & \leq\left\|\nabla\left(T^{-1}\right)\right\|_{\infty}^{n / p}\left(1+\|\nabla T\|_{\infty}^{p}\right)^{1 / p} \\
\left\|\left(T^{-1}\right)^{*}\right\|_{B\left(W^{1, p}(U), W^{1, p}(V)\right)} & \leq\|\nabla T\|_{\infty}^{n / p}\left(1+\left\|\nabla\left(T^{-1}\right)\right\|_{\infty}^{p}\right)^{1 / p} .
\end{aligned}
$$

Moreover, for $f \in W^{1, p}(V)$, the chain rule

$$
\nabla(f \circ T)=(\nabla f) \circ T \cdot \nabla T
$$

holds in $\mathcal{D}^{\prime}(U)$. 
Notice that the both sides of (4.14) are defined in the distribution sense, since $f \circ T \in L^{p}(U),(\nabla f) \circ T \in\left(L^{p}(U)\right)^{n}$ from Proposition 4.6 and $\nabla T$ is $L^{\infty}(U)$ valued matrix.

Proof. If $f \in C^{1}(V) \cap W^{1, p}(V)$, one can easily check by Corollary 4.2 that for almost every $x \in V$, the function $f \circ T(x)$ is totally differentiable at $x$. Thus (4.14) holds by the ordinary chain rule.

Next, let $f_{0} \in W^{1, p}(V)$. By the Meyers-Serrin theorem (see [A, Theorem 3.16]), there exists a sequence $\left\{f_{n}\right\} \subset C^{1}(V) \cap W^{1, p}(V)$ which approximates $f_{0}$ in $W^{1, p}(V)$. Then (4.14) holds for $f=f_{n}$. By Proposition 4.6, we have $f_{n} \circ T \rightarrow f_{0} \circ T$ in $L^{p}(U)$, and hence we have $\nabla\left(f_{n} \circ T\right) \rightarrow \nabla\left(f_{0} \circ T\right)$ in $\mathcal{D}^{\prime}(U)$. Moreover, since $\nabla f_{n} \rightarrow \nabla f_{0}$ in $L^{p}(V)$ and $\nabla T \in L^{\infty}(U)$, we have $\left(\nabla f_{n}\right) \circ T \cdot \nabla T \rightarrow\left(\nabla f_{0}\right) \circ T \cdot \nabla T$ in $L^{p}(U)$ by Proposition 4.6. Hence (4.14) holds for $f=f_{0}$.

By Proposition 4.6 and the chain rule (4.14), we have

$$
\begin{aligned}
& \left\|T^{*} f\right\|_{W^{1, p}(U)}^{p} \\
& \quad=\left\|T^{*} f\right\|_{L^{p}(U)}^{p}+\left\|T^{*} \nabla f \cdot \nabla T\right\|_{L^{p}(U)}^{p} \\
& \quad \leq\left\|\nabla\left(T^{-1}\right)\right\|_{\infty}^{n}\|f\|_{L^{p}(V)}^{p}+\left\|\nabla\left(T^{-1}\right)\right\|_{\infty}^{n}\|\nabla T\|_{\infty}^{p}\|\nabla f\|_{L^{p}(V)}^{p} .
\end{aligned}
$$

Thus the first equality of (4.13) holds. We can prove the second equality of (4.13) similarly.

\section{§4.3. Divergence formula}

In this subsection, we shall prove the divergence formula on Lipschitz domains. First, we shall remind the definition of the boundary integral. Note that the boundary $\partial \Omega$ of $\Omega \in L M(r, A, B)$ is a measurable space with the $\sigma$-field generated by the relative topology of $\boldsymbol{R}^{d}$.

Definition 4.3. Let $\left(\Omega,\left\{U_{k}, \chi_{k}, S_{k}, \phi_{k}\right\}_{k=1}^{K}\right) \in L M(r, A, B)$. By (ii) of Definition 1.6, $\Gamma_{k}=U_{k} \cap \partial \Omega$ is parameterized by $y^{\prime} \in S_{k}(r)$ by the relation

$$
\Gamma_{k} \ni x\left(y^{\prime}\right)=\chi_{k}^{-1}\left(y^{\prime}, \phi_{k}\left(y^{\prime}\right)\right) .
$$

For a nonnegative measurable function $f$ on $\Gamma_{k}$, define

$$
\int_{\Gamma_{k}} f d S:=\int_{S_{k}(r)} f\left(y^{\prime}\right) \sqrt{1+\left|\nabla \phi_{k}\left(y^{\prime}\right)\right|^{2}} d y^{\prime},
$$

where we abbreviate the function $y^{\prime} \mapsto f\left(x\left(y^{\prime}\right)\right)$ as $f\left(y^{\prime}\right)$. For a nonnegative measurable function $f$ on $\partial \Omega$, define $\int_{\partial \Omega} f d S:=\sum_{k=1}^{K} \int_{\Gamma_{k}} \alpha_{k} f$, where $\left\{\alpha_{k}\right\}_{k=1}^{K}$ 
is a partition of unity subordinate to the covering $\left\{U_{k}\right\}_{k=1}^{K}$ of $\partial \Omega$, i.e. $\alpha_{k} \in$ $C_{0}^{\infty}\left(U_{k}\right), 0 \leq \alpha_{k} \leq 1$ and $\sum \alpha_{k}=1$ near $\partial \Omega$.

One can prove that this definition of the integral is independent of the choice of $\left\{U_{k}, \chi_{k}, S_{k}, \phi_{k}\right\}$ and $\left\{\alpha_{k}\right\}$, as usual way (since we can use the change of variables formula and the chain rule proved in the previous section, there is no difficulty caused by the Lipschitz continuity of $\left.\phi_{k}\right)$. With this definition, we can introduce the space $L^{p}(\partial \Omega, d S)$ for $1 \leq p \leq \infty$.

Next, we shall define the divergence of $u$ and the unit normal vector on $\partial \Omega$.

Definition 4.4. Let $\left(\Omega,\left\{U_{k}, \chi_{k}, S_{k}, \phi_{k}\right\}_{k=1}^{K}\right) \in L M(r, A, B)$ for some $r, A, B>0$. For $\boldsymbol{u} \in\left(W^{1,1}(\Omega)\right)^{d}$, define the divergence of $\boldsymbol{u}$ by

$$
\operatorname{div} \boldsymbol{u}(x):=\sum_{k=1}^{K} \partial_{x_{k}} u_{k}(x) \in L^{1}(\Omega) .
$$

Define the unit outer normal vector $\boldsymbol{n} \in\left(L^{\infty}(\partial \Omega)\right)^{d}$ by

$$
\begin{aligned}
\boldsymbol{n}\left(\chi_{k}^{-1}\left(y^{\prime}\right)\right) & :=\nabla \chi_{k}^{-1} \cdot \boldsymbol{n}_{k}\left(y^{\prime}\right), \\
\boldsymbol{n}_{k}\left(y^{\prime}\right) & :=\frac{1}{\sqrt{1+\left|\nabla \phi_{k}\left(y^{\prime}\right)\right|^{2}}}\left(-\nabla \phi_{k}\left(y^{\prime}\right), 1\right) \in \boldsymbol{R}^{d},
\end{aligned}
$$

for $y^{\prime} \in S_{k}(r)$, where we use the parameterization (4.15).

One can easily check that the definition of $\boldsymbol{n}$ is independent of the choice of local coordinates.

We shall prove the divergence formula:

Proposition 4.8. Let $\left(\Omega,\left\{U_{k}, \chi_{k}, S_{k}, \phi_{k}\right\}_{k=1}^{K}\right) \in L M(r, A, B)$ for some $r, A, B>0$. Then, for $\boldsymbol{u} \in\left(W^{1,1}(\Omega)\right)^{d}$, we have $\left.\boldsymbol{u}\right|_{\partial \Omega} \in\left(L^{1}(\partial \Omega, d S)\right)^{d}$ and

$$
\int_{\Omega} \operatorname{div} \boldsymbol{u}(x) d x=\int_{\partial \Omega}{ }^{t} \boldsymbol{u} \cdot \boldsymbol{n} d S .
$$

Notice that the right hand side of (4.16) makes sense since $\left.\boldsymbol{u}\right|_{\partial \Omega} \in\left(L^{1}(\partial \Omega, d S)\right)^{d}$ and $\boldsymbol{n} \in\left(L^{\infty}(\partial \Omega)\right)^{d}$. We remark we can not use Proposition 3.2 for proving $\left.\boldsymbol{u}\right|_{\partial \Omega} \in\left(L^{1}(\partial \Omega, d S)\right)^{d}$, since in the proof of Proposition 3.2 we use the argument given in Section 5 of [D-I-M], in which we use the divergence formula.

Proof. By an argument using the partition of unity, we see that it is sufficient to show that for $\boldsymbol{u} \in\left(W^{1,1}\left(U_{k}^{-}\right)\right)^{d}$ with $\operatorname{supp} \boldsymbol{u} \cap \partial U_{k}^{-} \subset \Gamma_{k}$, the 
following statements

$$
\left.\boldsymbol{u}\right|_{\Gamma_{k}} \in\left(L^{1}\left(\Gamma_{k}, d S\right)\right)^{d}, \quad \int_{U_{k}^{-}} \operatorname{div} \boldsymbol{u}(x) d x=\int_{\Gamma_{k}}{ }^{t} \boldsymbol{u} \cdot \boldsymbol{n} d S,
$$

hold for fixed $k$. Moreover, we may assume $\chi_{k}=i d$ without loss of generality.

Take $\boldsymbol{u} \in\left(W^{1,1}\left(U_{k}^{-}\right)\right)^{d}$ with supp $\boldsymbol{u} \cap \partial U_{k}^{-} \subset \Gamma_{k}$. Since $\boldsymbol{u}(x) \in\left(W^{1,1}(\Omega)\right)^{d}$ and the map $\left(x^{\prime}, x_{d}\right)=\left(y^{\prime}, \phi_{k}\left(y^{\prime}\right)+y_{d}\right)$ is a Lipschitz homeomorphism, we have the function $y=\left(y^{\prime}, y_{d}\right) \mapsto \boldsymbol{u}\left(y^{\prime}, \phi_{k}\left(y^{\prime}\right)+y_{d}\right)$ belongs to $\left(W^{1,1}\left(S_{k}(r) \times(-r, 0)\right)\right)^{d}$ by Proposition 4.7 (we denote this function by the same letter $\boldsymbol{u}(y)$, by the abuse of notation). Moreover,

$$
\operatorname{supp} \boldsymbol{u}(y) \cap \partial\left(S_{k}(r) \times(-r, 0)\right) \subset S_{k}(r) \times\{0\} .
$$

Since $\left(y^{\prime}, y_{d}\right)=\left(x^{\prime},-\phi_{k}\left(x^{\prime}\right)+x_{d}\right)$, we have

$$
\begin{aligned}
\int_{U_{k}^{-}} \operatorname{div}_{x} \boldsymbol{u}(x) d x & =\int_{U_{k}^{-}} \sum_{l=1}^{d} \frac{\partial u_{l}}{\partial x_{l}}(x) d x \\
& =\int_{S_{k}(r) \times(-r, 0)}\left\{\sum_{l=1}^{d-1}\left(\frac{\partial u_{l}}{\partial y_{l}}-\frac{\partial \phi_{k}}{\partial y_{l}} \frac{\partial u_{l}}{\partial y_{d}}\right)(y)+\frac{\partial u_{d}}{\partial y_{d}}(y)\right\} d y
\end{aligned}
$$

where we used Corollary 4.5, $\operatorname{det}(\partial x / \partial y)=1$ and Proposition 4.7.

Since $\boldsymbol{u}(y) \in\left(W^{1,1}\left(S_{k}(r) \times(-r, 0)\right)\right)^{d}$, the function $y_{l} \mapsto \partial_{y_{l}} \boldsymbol{u}\left(y_{1}, \ldots, y_{l}\right.$, $\left.\ldots, y_{d}\right)$ is integrable for almost every $\hat{y}_{l}=\left(y_{1}, \ldots, y_{l-1}, y_{l+1}, \ldots, y_{d}\right)$. Hence the function $y_{l} \mapsto \boldsymbol{u}\left(y_{1}, \ldots, y_{l}, \ldots, y_{d}\right)$ is absolutely continuous for almost every $\hat{y_{l}}$. Since $S_{k}(r)$ is convex, the section

$$
I_{l}\left(\hat{y}_{l}\right)=\left\{y_{l} \mid y=\left(y_{l}, \hat{y}_{l}\right) \in S_{k}(r) \times(-r, 0)\right\}
$$

is an open interval $\left(s_{-}\left(\hat{y}_{l}\right), s_{+}\left(\hat{y}_{l}\right)\right)$. In particular, $s_{-}\left(\hat{y}_{d}\right)=-r, s_{+}\left(\hat{y}_{d}\right)=0$. Thus, we have

$$
\begin{aligned}
\int_{S_{k}(r) \times(-r, 0)} \partial_{y_{l}} \boldsymbol{u}\left(y_{l}, \hat{y}_{l}\right) d y & =\int_{\hat{P}_{l}\left(S_{k}(r) \times(-r, 0)\right)}\left\{\boldsymbol{u}\left(s_{+}\left(\hat{y}_{l}\right)\right)-\boldsymbol{u}\left(s_{-}\left(\hat{y}_{l}\right)\right)\right\} d \hat{y}_{l} \\
& = \begin{cases}0 & (l=1, \ldots, d-1) \\
\int_{S_{k}(r)} \boldsymbol{u}\left(y^{\prime}, 0\right) d y^{\prime} & (l=d)\end{cases}
\end{aligned}
$$

where $\hat{P}_{l}\left(S_{k}(r) \times(-r, 0)\right):=\left\{\hat{y}_{l} \mid\left(y_{l}, \hat{y}_{l}\right) \in S_{k}(r) \times(-r, 0)\right.$ for some $\left.y_{l}\right\}$. We use (4.18) and put $y^{\prime}=\hat{y}_{d}$. The integral in the last member of (4.20) converges, 
since

$$
\int_{S_{k}(r)}\left|\boldsymbol{u}\left(y^{\prime}, 0\right)\right| d y^{\prime} \leq \int_{S_{k}(r)} \int_{-r}^{0}\left|\partial_{y_{d}} \boldsymbol{u}\right| d y_{d} d y^{\prime} \leq\|\nabla \boldsymbol{u}\|_{\left(L^{1}\left(S_{k}(r) \times(-r, 0)\right)\right)^{d}}<\infty,
$$

where we used $\boldsymbol{u}(y) \in\left(W^{1,1}\left(S_{k}(r) \times(-r, 0)\right)\right)^{d}$. Thus, $\boldsymbol{u}\left(y^{\prime}, 0\right) \in L^{1}\left(S_{k}(r)\right)$. Moreover,

$$
\int_{\Gamma_{k}}|\boldsymbol{u}| d S=\int_{S_{k}(r)}\left|\boldsymbol{u}\left(y^{\prime}, 0\right)\right| \sqrt{1+\left|\nabla \phi_{k}\right|^{2}} d y^{\prime} \leq \sqrt{1+A^{2}} \int_{S_{k}(r)}\left|\boldsymbol{u}\left(y^{\prime}, 0\right)\right| d y^{\prime},
$$

the restriction $\left.\boldsymbol{u}\right|_{\Gamma_{k}} \in\left(L^{1}\left(\Gamma_{k}, d S\right)\right)^{d}$.

Thus, we obtain from (4.19) and (4.20)

$$
\begin{aligned}
\int_{U_{k}^{-}} \operatorname{div}_{x} \boldsymbol{u}(x) d x & =\int_{S_{k}(r)}\left\{\int_{-r}^{0}\left(\sum_{l=1}^{d-1}-\frac{\partial \phi_{k}\left(y^{\prime}\right)}{\partial y_{l}} \frac{\partial u_{l}}{\partial y_{d}}+\frac{\partial u_{d}}{\partial y_{d}}\right) d y_{d}\right\} d y^{\prime} \\
& =\int_{S_{k}(r)}\left\{-\sum_{l=1}^{d-1} \frac{\partial \phi_{k}\left(y^{\prime}\right)}{\partial y_{l}} u_{l}\left(y^{\prime}\right)+u_{d}\left(y^{\prime}\right)\right\} d y^{\prime} \\
& =\int_{S_{k}(r)}{ }^{t} \boldsymbol{u}\left(y^{\prime}\right) \cdot \boldsymbol{n}_{k}\left(y^{\prime}\right) \sqrt{1+\left|\nabla \phi_{k}\left(y^{\prime}\right)\right|^{2}} d y^{\prime} \\
& =\int_{\Gamma_{k}}{ }^{t} \boldsymbol{u} \cdot \boldsymbol{n} d S .
\end{aligned}
$$

Hence we obtain (4.17).

\section{Acknowledgements}

We would like to thank Akira Iwatsuka for many helpful comments on all subjects. We would like to thank Shin-ichi Doi for the suggestion at the Analysis Seminar in Tsukuba University, by which we get the opportunity to research subjects in the present paper. We would also like to thank Kenji Yajima and Shu Nakamura for helpful comments at the Functional Analysis Seminar in Tokyo University, by which we improve the proof of Proposition 3.2 .

\section{References}

[A] Adams, R. A., Sobolev Spaces, Academic Press, 1978.

[C-F-K-S] Cycon, H. L., Froese, R. G., Kirsch, W. and Simon, B., Schrödinger Operators with Applications to Quantum Mechanics and Global Geometry, SpringerVerlag, 1987. 
[Cv] Yves Colin de Verdiere, L'asymptotique de Weyl pour les bouteilles magnétiques, Comm. Math. Phys., 105 (1986), 327-335.

[D-I-M] Doi, S., Iwatsuka, A. and Mine, T., The uniqueness of the integrated density of states for the Schrödinger operators with magnetic fields, Math. Z., 237 (2001), $335-371$.

[H-L-M-W1] Hupfer, T., Leschke, H., Müller, P. and Warzel, S., The absolute continuity of the integrated density of states for magnetic Schrödinger operators with certain unbounded random potentials, Comm. Math. Phys., 221 (2001), 229-254.

[H-L-M-W2] - Existence and uniqueness of the integrated density of states for Schrödinger operators with magnetic fields and unbounded random potentials, preprint, to appear in Rev. Math. Phys., e-print math-ph/0010013 (2000).

[I-K] Ikebe, T. and Kato, T., Uniqueness of the self-adjoint extension of singular elliptic differential operators, Arch. Rational Mech. Anal., 9 (1962), 77-92.

[K] Kato, T., Perturbation Theory for Linear Operators, Springer-Verlag, 1966.

[L-S] Leinfelder, H. and Simadar, C. G., Schrödinger operators with singular magnetic vector potentials, Math. Z., 176 (1981), 1-19.

[N] Nakamura, S., A remark on the Dirichlet-Neumann decoupling and the integrated density of states, J. Funct. Anal., 179 (2001), 136-152.

[Ra] Rademacher, H., Über partielle und totale Differenzierbarkeit von funktionen mehrerer Variabeln und über die Transformation der Doppelintegrale, Math. Ann., 79 (1919), 340-359.

[Ru1] Rudin, W., Real and Complex Analysis, McGraw-Hill, 1987.

[Ru2] —- Functional Analysis; second edition, McGraw-Hill, 1991.

[Wl] Wloka, J., Partial Differential Equations, Cambridge Univ. Press, 1987. 